\title{
FROM HARMONIC ANALYSIS TO ARITHMETIC COMBINATORICS
}

\author{
IZABELLA ŁABA
}

Arithmetic combinatorics, or additive combinatorics, is a fast developing area of research combining elements of number theory, combinatorics, harmonic analysis and ergodic theory. Its arguably best-known result, and the one that brought it to global prominence, is the proof by Ben Green and Terence Tao of the long-standing conjecture that primes contain arbitrarily long arithmetic progressions. There are many accounts and expositions of the Green-Tao theorem, including the articles by Kra [119] and Tao [182] in the Bulletin.

The purpose of the present article is to survey a broader, highly interconnected network of questions and results, built over the decades and spanning several areas of mathematics, of which the Green-Tao theorem is a famous descendant. An old geometric problem lies at the heart of key conjectures in harmonic analysis. A major result in partial differential equations invokes combinatorial theorems on intersecting lines and circles. An unexpected argument points harmonic analysts towards additive number theory, with consequences that could have hardly been anticipated.

We will not try to give a comprehensive survey of harmonic analysis, combinatorics, or additive number theory. We will not even be able to do full justice to our specific areas of focus, instead referring the reader to the more complete expositions and surveys listed in Section 7. Our goal here is to emphasize the connections between these areas; we will thus concentrate on relatively few problems, chosen as much for their importance to their fields as for their links to each other. The article is written from the point of view of an analyst who, in the course of her work, was gradually introduced to the questions discussed here and found them fascinating. We hope that the reader will enjoy a taste of this experience.

\section{The Kakeya PRoblem}

1.1. Life during wartime. By all accounts, Abram Samoilovitch Besicovitch (1891-1970) had an interesting life. He was born in Berdyansk, in the south of

Received by the editors May 28, 2007.

2000 Mathematics Subject Classification. Primary 11B25, 11B75, 11L07, 28A75, 28A78, 42B15, 42B20, 42B25, 52C10.

This article is based on lectures presented at the Winter 2004 meeting of the Canadian Mathematical Society, Montreal, December 2004; the MSRI workshop "Women in Mathematics: The Legacy of Ladyzhenskaya and Oleinik", Berkeley, May 2006; the Fall 2006 Western Section meeting of the American Mathematical Society, Salt Lake City, October 2006; the AMS Current Events Bulletin Session, Joint Mathematics Meetings, New Orleans, January 2007; and the Pennsylvania State University, State College, April 2007.

The author is supported in part by an NSERC Discovery Grant. 
Russia. Having demonstrated exceptional mathematical abilities at an early age, he went on to study under the direction of the famous probabilist A.A. Markov at the University of St. Petersburg, from which he graduated in 1912.

The University of Perm was established in October 1916, first as a branch of the University of St. Petersburg and then as an independent institution. Perm, located in the Ural Mountains, was closed off to foreign visitors from the 1920s until 1989, and the university, which remains the main intellectual center of the region, has seen difficult times. But in the hopeful early years, from 1916 to about 1922, it managed to attract many brilliant and ambitious young academics. Besicovitch was appointed professor of mathematics at the University of Perm in 1917. Among his colleagues were the mathematician I.M. Vinogradov, of the three-primes theorem in analytic number theory, and the physicist A.A. Friedmann, best known for his mathematical models of the "big bang" and the expanding universe.

After several months of political unrest, the Bolshevik Revolution erupted in October 1917. Soon thereafter a civil war engulfed Russia. The White Army, led by former Tsarist officers, opposed the communist Red Army. Perm was controlled by the Red Army until December 1918, when the White Army took over. In August 1919 the Red Army returned. According to Friedmann, all the staff except Besicovitch left the university:

The only person who kept his head and saved the remaining property was Besicovitch, who is apparently A.A. Markov's disciple not only in mathematics but also with regard to resolute, precise definite actions.

In 1920 Besicovitch returned to St. Petersburg, which had been renamed Petrograd six years earlier, and accepted a position at Petrograd University. (Petrograd would change names twice more: it became Leningrad after Lenin's death in 1924, and in 1991 it reverted to its original name, St. Petersburg.) The war years had not been kind to Petrograd. The city lost its capital status to Moscow in 1918, the population dwindled to a third of its former size, and the economy was in tatters. This is how Encyclopedia Britannica describes the education reform in the newborn Soviet Union:

To destroy what they considered the elitist character of Russia's educational system, the communists carried out revolutionary changes in its structure and curriculum. All schools, from the lowest to the highest, were nationalized and placed in charge of the Commissariat of Enlightenment. Teachers lost the authority to enforce discipline in the classroom. Open admission to institutions of higher learning was introduced to assure that anyone who desired, regardless of qualifications, could enroll. Tenure for university professors was abolished, and the universities lost their traditional right of selfgovernment.

Besicovitch was awarded a Rockefeller Fellowship in 1924 but was denied permission to leave Russia. He escaped illegally, along with his colleague J.D. Tamarkin, and took up his fellowship in Copenhagen, working with Harald Bohr. After a brief stay in Liverpool (1926-27), he finally settled down in Cambridge, where he spent the rest of his life. From 1950 until his retirement in 1958, he was the Rouse Ball Professor of Mathematics; this is the same chair that was held by John Littlewood 
prior to Besicovitch's tenure and is currently being held by W.T. Gowers, whose work will play a major part later in this story.

Besicovitch will be remembered for his contributions to the theory of almost periodic functions (a subject to which Bohr introduced him in Copenhagen) and other areas of function theory, and especially for his pioneering work in geometric measure theory, where he established many of the fundamental results. He was a powerful problem solver who combined a mastery of weaving long and intricate arguments with a capacity to approach a question from unexpected angles. His solution of the Kakeya problem, to which we are about to turn, is a prime example of his ingenuity.

1.2. Riemann integrals and rotating needles. In the midst of the civil war, between the alternating takeovers of Perm by the Red and White Armies, Besicovitch was working on a problem in Riemann integration:

Given a Riemann-integrable function $f$ on $\mathbb{R}^{2}$, must there exist a rectangular coordinate system $(x, y)$ such that $f(x, y)$ is a Riemannintegrable function of $x$ for each fixed $y$, and the two-dimensional integral of $f$ is equal to the iterated integral $\iint f(x, y) d x d y$ ?

He observed that to answer the question in the negative it would suffice to construct a set of zero Lebesgue measure in $\mathbb{R}^{2}$ containing a line segment in every direction. Specifically, suppose that $E$ is such a set, and fix a coordinate system in $\mathbb{R}^{2}$. Let $f$ be defined so that $f(x, y)=1$ if $(x, y) \in E$ and if at least one of $x, y$ is rational, and $f(x, y)=0$ otherwise. We may also assume, shifting $E$ if necessary, that the $x$ - and $y$-coordinates of the line segments parallel to the $y$ - and $x$-axes, respectively, are irrational. Then for every direction in $\mathbb{R}^{2}$, there is at least one line segment in that direction along which $f$ is not Riemann-integrable as a function of one variable. However, $f$ is Riemann-integrable in two dimensions, as the set of its points of discontinuity has planar measure 0 .

Besicovitch then proceeded to construct the requisite set $E$. This, along with the solution of the Riemann integration problem, was published in a Perm scientific journal in 1919 [4].

The idea is roughly as follows. Fix a triangle $A B C$ and observe that it contains line segments of all slopes between those of $A B$ and $A C$. Next, cut it into many long and thin triangles with one vertex at $A$ and the other two on the base line segment $B C$, then rearrange these triangles by sliding them along the base as in Figure 1. The new set still contains line segments of all slopes as noted above; on the other hand, given $\epsilon>0$, we can arrange for the set to have area less than $\epsilon$ by making sufficiently many cuts and optimizing the configuration of the small triangles. To produce a set of measure 0 , still containing line segments of all slopes between those of $A B$ and $A C$, iterate the construction and then take the limit. Finally, a union of finitely many rotated copies of the set just constructed will have measure 0 and contain a line segment in every direction. The details can be found in many references, including Davis-Chang [42], Falconer [58], Stein [169], and Wolff [193].

Besicovitch's original construction was subsequently simplified by other authors, notably Perron and Schoenberg. Several alternative constructions based on different ideas were found later, including one due to Kahane [105] and another due to Besicovitch himself [7]. 

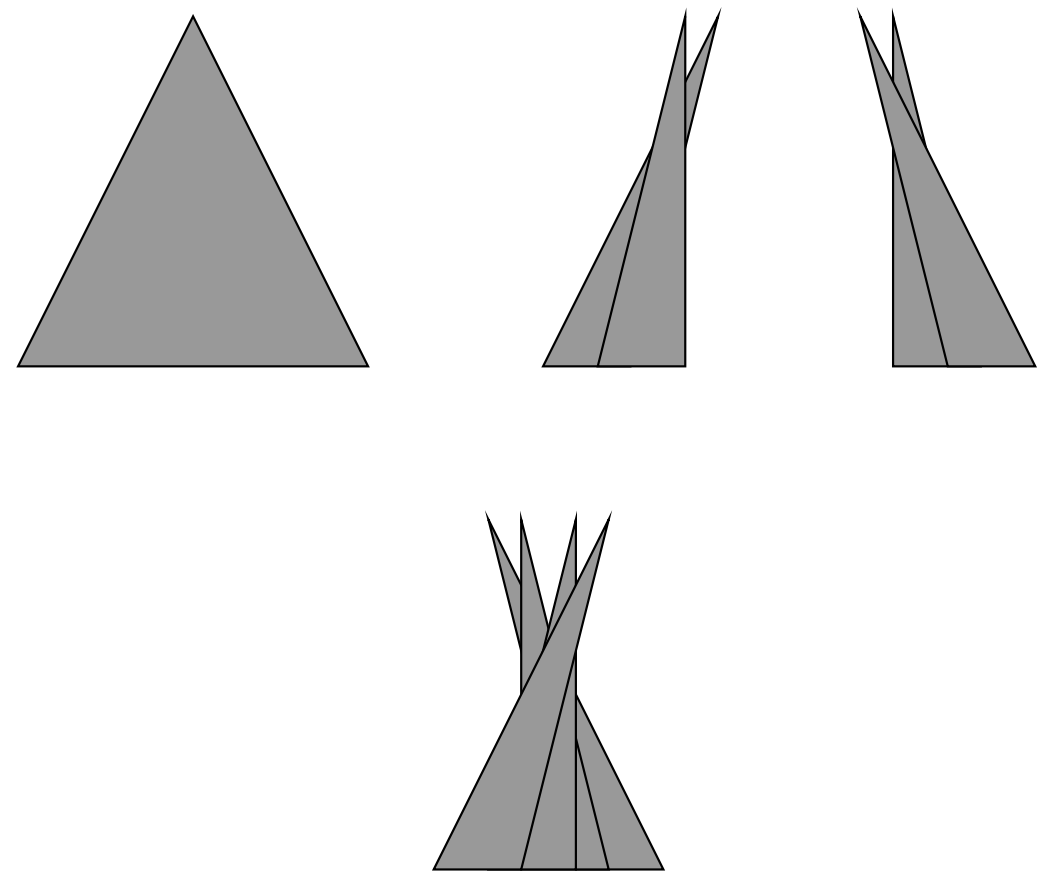

Figure 1. The first two steps of a Besicovitch set construction.

Due to the civil war, there was hardly any scientific communication between Russia and the Western world at the time. Besicovitch was thus unaware that a similar question, now known as the Kakeya needle problem, was independently proposed by the Japanese mathematician Soichi Kakeya around the same time:

What is the smallest area of a planar region within which a unit line segment (a "needle") can be rotated continuously through 180 degrees, returning to its original position but with reversed orientation?

Kakeya [106] and Fujiwara-Kakeya [65] conjectured that the smallest convex planar set with this property was the equilateral triangle of height 1 and mentioned that one could do better if the convexity assumption was dropped. For example, the region bounded by a three-cusped hypocycloid inscribed in a circle of radius 1 has the required property and has area $\pi / 8 \approx .39$, whereas the area of the triangle is $\sqrt{3} / 3 \approx 0.58$. Kakeya's conjecture for the convex case was soon confirmed by Julius Pál [142], but the more interesting non-convex problem remained open. Besicovitch learned of Kakeya's problem after he left Russia, possibly from a 1925 book by G.D. Birkhoff which he cites in [6], and realized that a modification of his earlier construction (together with an additional trick due to Pál) provided the unexpected answer:

For any $\epsilon>0$, there is a planar region of area less then $\epsilon$ within which a needle can be rotated through 180 degrees. 
His solution was published in 1928 [5]. There are now many other such constructions, some with additional constraints on the planar region in question. Cunningham [40] rotates a needle within a simply connected region, contained in a unit disc, of area smaller than any $\epsilon>0$.

\subsection{The Kakeya conjecture.}

Definition 1.1. A Kakeya set, or a Besicovitch set, is a subset of $\mathbb{R}^{d}$ which contains a unit line segment in each direction.

Besicovitch's construction shows that Kakeya sets in dimension 2 can have measure 0 . With this information, it is easy to deduce a similar result in higher dimensions: if $E$ is a planar Kakeya set of measure 0 , then the set $E \times[0,1]^{d-2}$ in $\mathbb{R}^{d}$ is a Kakeya set and has $d$-dimensional measure 0 . It turns out, however, that many problems in analysis call for more detailed information on the size of Kakeya sets in terms of their dimension. There are several non-equivalent formulations of the problem, the best known of which is as follows.

Conjecture 1.2. A Kakeya set in $\mathbb{R}^{d}$ must have Hausdorff dimension d.

We will use $\operatorname{dim}_{H}(E)$ to denote the Hausdorff dimension of a set $E$. We will not define it here; the interested reader will find the definition and background information in Falconer [58] or Mattila [127]. For the purpose of this exposition, it will be easier to work instead with an alternative formulation in terms of the Minkowski dimension (also known as the box dimension).

For a compact set $E \subset \mathbb{R}^{d}$, let $E_{\delta}$ be the $\delta$-neighbourhood of $E$, and consider the asymptotic behaviour of the $d$-dimensional volume of $E_{\delta}$ as $\delta \rightarrow 0$. The upper and lower Minkowski dimensions of $E$ are given by

$$
\begin{aligned}
& \overline{\operatorname{dim}}_{M}(E)=\inf \left\{\alpha \in[0, d]:\left|E_{\delta}\right| \leq C_{\alpha} \delta^{d-\alpha}\right\}, \\
& \underline{\operatorname{dim}}_{M}(E)=\sup \left\{\alpha \in[0, d]:\left|E_{\delta}\right| \geq C_{\alpha} \delta^{d-\alpha}\right\},
\end{aligned}
$$

with the constants $C_{\alpha}$ uniform in $\delta$. We will say that $E$ has Minkowski dimension $\beta$ if $\underline{\operatorname{dim}}_{M}(E)=\overline{\operatorname{dim}}_{M}(E)=\beta$.

Conjecture 1.3. A Kakeya set in $\mathbb{R}^{d}$ must have Minkowski dimension d.

We have

$$
\operatorname{dim}_{H}(E) \leq \underline{\operatorname{dim}}_{M}(E) \leq \overline{\operatorname{dim}}_{M}(E)
$$

for all compact sets $E \subset \mathbb{R}^{d}$. There are sets for which the inequalities are sharp: for instance, any countable set has Hausdorff dimension 0, but we invite the reader to verify that the set $\left\{1, \frac{1}{2}, \frac{1}{3}, \ldots\right\}$ has Minkowski dimension $1 / 2$. Thus Conjecture 1.3 is formally weaker than Conjecture 1.2. Similarly, any lower bound on the Hausdorff dimension of Kakeya sets implies the same bound on the Minkowski dimension, but the converse implication does not hold.

The Kakeya conjecture has been fully resolved in dimension 2. Davies [41] proved that a Kakeya set $E$ in $\mathbb{R}^{2}$ must have Hausdorff dimension 2; a more robust argument due to Córdoba [38] gives the optimal quantitative estimate $\left|E_{\delta}\right| \geq\left(\log \left(\frac{1}{\delta}\right)\right)^{-1}$. The problem remains open for all $d \geq 3$.

The Kakeya problem has been linked to surprisingly diverse questions in several areas of mathematics. The current interest in it is motivated by applications to harmonic analysis and partial differential equations. On the other hand, the partial 
results to date have relied on a variety of geometric, combinatorial and numbertheoretic methods. While this has resulted in substantial progress, most experts believe that we are still quite far from resolving the problem.

\section{QUESTIONS IN HARMONIC ANALYSIS}

2.1. Maximal functions and averaging estimates. Averaging and maximal estimates in analysis go back at least as far as the Lebesgue differentiation theorem:

Theorem 2.1. If $f: \mathbb{R}^{d} \rightarrow \mathbb{R}$ is integrable, then for almost all $x$ we have

$$
\lim _{r \rightarrow 0} \frac{1}{|B(x, r)|} \int_{B(x, r)} f(y) d y=f(x),
$$

where $B(x, r)=\{y:|x-y| \leq r\}$.

Amazing variations can be spun on this very basic result if we allow averages over more complicated sets rather than balls to be considered, but first, two remarks are in order. One concerns the geometric content of the theorem: if $f=\chi_{E}$ is the characteristic function of a measurable set $E,(2.1)$ states that for almost all $x \in E$

$$
\lim _{r \rightarrow 0} \frac{|E \cap B(x, r)|}{|B(x, r)|}=1,
$$

which is the Lebesgue theorem on density points. The second remark is that Theorem 2.1 can be deduced, with very little additional effort, from a maximal function estimate due to Hardy-Littlewood [93] in dimension 1 and to Wiener [192] in higher dimensions. Given $f \in L^{1}\left(\mathbb{R}^{d}\right)$, define its Hardy-Littlewood maximal function by

$$
M f(x)=\sup _{r>0} \frac{1}{|B(x, r)|} \int_{B(x, r)}|f(y)| d y .
$$

Then $\|M f\|_{p} \leq C_{p, d}\|f\|_{p}$ for all $1<p \leq \infty$, and $M$ is of weak type (1,1) (for the non-specialist, this is just slightly weaker than boundedness on $L^{1}\left(\mathbb{R}^{d}\right)$ ).

The balls in (2.1)-(2.3) can be harmlessly replaced by cubes, equilateral symplices, or similar images of any fixed $d$-dimensional solid. This is easy and somewhat unexciting. Consider, though, a "lower-dimensional" Nikodym-type maximal function on $\mathbb{R}^{2}$,

$$
M^{N} f(x)=\sup _{\ell \ni x} \int_{\ell}|f(y)| d \sigma_{\ell}(y),
$$

where $\ell$ ranges over all straight lines through a fixed $x$, and $\sigma_{\ell}$ is the 1-dimensional Lebesgue measure on $\ell$. The operator $M^{N}$ cannot be bounded on any $L^{p}$ space, due to a result of Nikodym [136]:

There is a set $N \subset[0,1]^{2}$ of planar Lebesgue measure 1 such that for every $x \in N$ there is a line $\ell_{x}$ such that $N \cap \ell_{x}=\{x\}$.

If $f$ is the characteristic function of the complement of $N$ in $[0,1]^{2}$, we have $f \equiv 0$ almost everywhere, yet $M^{N} f(x) \geq \frac{2 \sqrt{2}}{3}$ for all $x \in N \cap\left[\frac{1}{3}, \frac{2}{3}\right]^{2}$. The same example shows that there is no analogue of Theorem 2.1 with balls replaced by lines. We're not in Kansas anymore.

Maximal operators such as

$$
M f(x)=\sup _{T \ni x} \int_{T}|f(y)| d \sigma_{T}(y)
$$


and similarly defined averaging operators, where $T$ ranges over "thin" objects in a specified class and $\sigma_{T}$ is a probabilistic measure on $T$, have been attracting much interest in contemporary harmonic analysis. The x-ray and Radon transforms, widely used in medical imaging and other applied problems, involve averages over lowerdimensional affine subspaces of $\mathbb{R}^{d}$; see e.g. Oberlin-Stein [137], Drury [43], Christ [34], and Bourgain [13]. Averages over curved submanifolds have found numerous applications to Fourier integral operators and PDE theory, e.g. Christ et al. [36], Mockenhaupt-Seeger-Sogge [130], and Phong-Stein [143], [144]. A broad overview of this area of research and many other references can be found in monographs such as Stein [169].

The regularity properties ( $L^{p}$ boundedness, Sobolev regularity, mixed norm estimates) of averaging and maximal operators such as (2.4) depend on both analytic and combinatorial-geometric properties of the $T$-objects under consideration. The traditional approach is analytic, relying on properties such as smoothness and curvature. The combinatorial-geometric considerations took longer to claim their share of the spotlight. As in the Nikodym example, if many $T$-objects can be packed into a set of small size, poor regularity properties are to be expected. Positive regularity results call for a converse implication, which is usually deeper and more difficult to prove.

Analysts quickly realized that Besicovitch's construction of Kakeya sets of measure zero, as well as Nikodym's result stated above (the two results were later shown to be closely related), could be used to produce counterintuitive examples involving maximal functions and differentiation of integrals. In particular, the maximal function

$$
M^{*} f(x)=\sup _{R \ni x} \frac{1}{|R|} \int_{R}|f(y)| d y,
$$

where $R$ ranges over all rectangular boxes containing $x$, is not bounded on $L^{p}\left(\mathbb{R}^{d}\right)$ for any finite $p$ if $d \geq 2$; similarly, there is no analogue of Theorem 2.1 with balls replaced by rectangular boxes of arbitrary proportions and orientation. This was observed in Nikodym [136] and Busemann-Feller [28].

Returning to the Nikodym maximal function example, it would not do to abandon it altogether. Nikodym and Kakeya-based constructions are too ubiquitous in multidimensional harmonic analysis. Instead, the Nikodym maximal function is usually defined as

$$
M_{\delta}^{N} f(x)=\sup _{T_{\delta} \ni x} \frac{1}{\left|T_{\delta}\right|} \int_{T_{\delta}}|f(y)| d y,
$$

where $\delta>0$ is a small parameter, and the averages are taken over all rectangles (or tubes, in higher dimensions) $T_{\delta}$ of fixed length 1 and diameter $\delta$. Now a reasonable course of action is to try to prove bounds on $M_{\delta}^{N}$ in terms of $\delta$ and use them just as we would, in an alternate universe without Kakeya or Nikodym sets, use the boundedness of the maximal averages over lines.

The Kakeya conjecture is closely related to $L^{p}$ bounds on the Kakeya twin of $M_{\delta}^{N}$ :

$$
M_{\delta}^{K} f(u)=\sup _{T_{\delta}} \frac{1}{\left|T_{\delta}\right|} \int_{T_{\delta}}|f(y)| d y,
$$

where $u \in S^{d-1}$ is a direction, $\delta>0$ is a small parameter as above, and $T_{\delta}$ ranges over all tubes of length 1 and diameter $\delta$ whose long direction is parallel to $u$. This 
maximal function was first explicitly defined by Jean Bourgain [13], who conjectured that the estimate

$$
\forall \epsilon>0, \quad\left\|M_{\delta}^{K} f\right\|_{L^{d}\left(S^{d-1}\right)} \leq C_{\epsilon} \delta^{\epsilon}\|f\|_{L^{d}\left(\mathbb{R}^{d}\right)}
$$

should hold. (2.6), formally slightly stronger than Conjecture 1.2, follows from several outstanding conjectures in harmonic analysis and partial differential equations, including the Bochner-Riesz conjecture on the spherical summation of the Fourier series of $L^{p}$ functions, Sogge's local smoothing conjecture on the spacetime $L^{p}$ regularity of solutions to the wave equation [158], and the restriction conjecture, to which we will turn shortly. The converse implication is not known, but harmonic analysts believe that proving (2.6) would be a major step towards proving these conjectures.

A classic example of a maximal theorem over hypersurfaces in $\mathbb{R}^{d}$ is the spherical maximal theorem, due to E.M. Stein [167] for $d \geq 3$ and Bourgain [11] for $d=2$.

Theorem 2.2. Define the spherical maximal operator in $\mathbb{R}^{d}$ by

$$
M^{S} f(x)=\sup _{t>0} \int_{S^{d-1}}|f(x-t y)| d \sigma(y)
$$

where $\sigma$ is the normalized Lebesgue measure on $S^{d-1}$. Then

$$
\left\|M^{S} f(x)\right\|_{L^{p}\left(\mathbb{R}^{d}\right)} \leq C\|f\|_{L^{p}\left(\mathbb{R}^{d}\right)}, p>\frac{d}{d-1},
$$

and this range of $p$ is optimal.

This implies a differentiation theorem for spheres. Let

$$
A_{t} f(x)=\int_{S^{d-1}} f(x-t y) d \sigma(y)
$$

then for all $f \in L^{p}\left(\mathbb{R}^{d}\right), p>\frac{d}{d-1}$, we have $\lim _{t \rightarrow 0} A_{t} f(x)=f(x)$ for almost all $x \in \mathbb{R}^{d}$. We also note the corresponding geometric statement in $\mathbb{R}^{2}$, proved by Bourgain [11] via Theorem 2.2 and, independently, by Marstrand [124], who used a purely geometric argument.

Corollary 2.3. Let $E \subset \mathbb{R}^{2}$. Suppose that for every $x \in \mathbb{R}^{2} E$ contains a circle centered at $x$. Then $E$ must have positive 2-dimensional Lebesgue measure.

It might not be clear at first sight that estimates such as (2.8) have any relevance to regularity results for partial differential equations. Recall, however, that the Cauchy problem for the wave equation in $\mathbb{R}_{x}^{3} \times \mathbb{R}_{t}$,

$$
u_{t t}-\Delta_{x} u=0, u(x, 0)=f(x), u_{t}(x, 0)=g(x),
$$

is solved by the well-known explicit formula in terms of spherical averages,

$$
u(x, t)=\frac{\partial}{\partial t}\left(t A_{t} f(x)\right)+t A_{t} g(x) .
$$

This connection could take us very far into PDE theory (see e.g. MockenhauptSeeger-Sogge [130], Sogge [157], and Tao [181]); we will return to it briefly in Section 4.2 , but will not pursue it here otherwise. 
2.2. The restriction problem. The Fourier transform of a function $f: \mathbb{R}^{d} \rightarrow \mathbb{C}$ is defined by

$$
\widehat{f}(\xi)=\int f(x) e^{-2 \pi i x \cdot \xi} d x
$$

This maps the Schwartz space of functions $\mathcal{S}$ to itself and is clearly a bounded operator from $L^{1}\left(\mathbb{R}^{d}\right)$ to $L^{\infty}\left(\mathbb{R}^{d}\right)$. A basic result in harmonic analysis is that the Fourier transform extends to an isometry on $L^{2}\left(\mathbb{R}^{d}\right)$. By the Hausdorff-Young inequality, it also extends to a bounded operator from $L^{p}\left(\mathbb{R}^{d}\right)$ to $L^{p^{\prime}}\left(\mathbb{R}^{d}\right)$ if $1<$ $p<2$ and $\frac{1}{p}+\frac{1}{p^{\prime}}=1$.

The following question has become known as the restriction problem:

Let $\mu$ be a non-zero measure on $\mathbb{R}^{d}$. For what values of $p^{\prime}, q^{\prime}$ does the Fourier transform, defined on $\mathcal{S}$, extend to a bounded operator from $L^{q^{\prime}}\left(\mathbb{R}^{d}\right)$ to $L^{p^{\prime}}\left(\mathbb{R}^{d}, d \mu\right)$ ? In other words, when do we have an estimate

$$
\|\widehat{f}\|_{L^{p^{\prime}}\left(\mathbb{R}^{d}, d \mu\right)} \leq C\|f\|_{L^{q^{\prime}}\left(\mathbb{R}^{d}\right)}, f \in \mathcal{S} ?
$$

We will usually assume that the measure $\mu$ is finite. Here and below, $C$ and other constants may depend on the dimension $d$, the measure $\mu$, and the exponents $p, q, p^{\prime}, q^{\prime}$, but not on $f$ except where explicitly indicated otherwise. Whenever we use the notation $L^{p}(X)$ without indicating the measure, the latter is assumed to be the Lebesgue measure on $X$.

In the classical version of the problem, $\mu$ is the Lebesgue measure on a $d-1$ dimensional hypersurface $\Gamma$ in $\mathbb{R}^{d}$, e.g. a sphere or a cone. The above question can then be rephrased in terms of restricting the Fourier transform of an $L^{q^{\prime}}$ function $f$ to the hypersurface. This is trivial if $q^{\prime}=1$ and $p^{\prime}=\infty$, since then $\widehat{f}$ is continuous and bounded everywhere, in particular on $\Gamma$. On the other hand, it is easy to see that no such result is possible if $q^{\prime}=2$. This is because the Fourier transform maps $L^{2}$ onto $L^{2}$, so that we are not able to say anything about the behaviour of $\widehat{f}$ on a set of measure 0. It is less clear what happens for $q^{\prime} \in(1,2)$. As it turns out, the answer here depends on the geometry of $\Gamma$ : for example, there can be no estimates such as (2.9) with $q^{\prime}>1$ if $\Gamma$ is a hyperplane, but we do have non-trivial restriction estimates for a variety of curved hypersurfaces such as a sphere or a cone.

The reason for the somewhat curious notation so far is that we reserved the exponents $p, q$ for the dual formulation of the problem. We will write $\widehat{f d \mu}(\xi)=$ $\int f(x) e^{-2 \pi i x \cdot \xi} d \mu(x)$.

Let $\mu$ be a non-zero measure on $\mathbb{R}^{d}$. For what values of $p, q$ do we have an estimate

$$
\|\widehat{f d \mu}\|_{L^{q}\left(\mathbb{R}^{d}\right)} \leq C\|f\|_{L^{p}\left(\mathbb{R}^{d}, d \mu\right)}, f \in \mathcal{S} ?
$$

A reasonably simple argument shows that (2.10) and (2.9) are equivalent if $p, p^{\prime}$ and $q, q^{\prime}$ are pairs of dual exponents: $\frac{1}{p}+\frac{1}{p^{\prime}}=\frac{1}{q}+\frac{1}{q^{\prime}}=1$. While the restriction problem took its name from the first formulation (2.9), the second one turns out to be much more useful in applications.

In the case when $\mu$ is the surface measure on a hypersurface $\Gamma$ with non-vanishing Gaussian curvature, classical stationary phase estimates (e.g. Herz [96]) yield asymptotic expressions for $\widehat{f d \mu}(\xi)$ if $f$ is a smooth compactly supported function 
on $\Gamma$. In particular, we then have

$$
|\widehat{f d \mu}(\xi)|=O\left((1+|\xi|)^{-\frac{d-1}{2}}\right),
$$

and it follows that $\widehat{f d \mu} \in L^{q}\left(\mathbb{R}^{d}\right)$ for $q>\frac{2 d}{d-1}$. A wide variety of similar estimates has been obtained under weaker assumptions on the curvature of $\Gamma$, for example "finite type" surfaces and surfaces with less than $d-1$ non-vanishing principal curvatures are allowed. A comprehensive survey of such work up to 1993 is given in Stein [169] (see also Hörmander [97]).

The point of restriction estimates is that the smoothness conditions on $f$ can be replaced by the weaker assumption that $f \in L^{p}\left(\mathbb{R}^{d}, d \mu\right)$, provided that we are willing to trade pointwise estimates on $\widehat{f d \mu}$ such as (2.11) for $L^{q}$ bounds with appropriate $q=q(p)$. This is useful in applications to partial differential equations, where restriction techniques can yield $L^{q}$ or mixed-norm regularity of solutions if the initial data is only known to be in some $L^{p}$ space. For example, restriction estimates are very closely related to Strichartz estimates [170]. Restriction estimates are also relevant to many other problems in harmonic analysis, including oscillatory integrals, singular integrals, averaging and maximal operators, and Fourier integral operators. We will not attempt to survey this rich and complex area here, instead encouraging the reader to consult references such as Sogge [157], Stein [169], Tao [177], [181], and Wolff [200].

2.3. Restriction for the sphere and arrangements of needles. We will now take a closer look at the restriction phenomenon for the unit sphere $S^{d-1}$ in $\mathbb{R}^{d}$. Let $\sigma$ be the normalized surface measure on $S^{d-1}$. The following conjecture is due to Elias M. Stein [168]:

Conjecture 2.4. For all $q>\frac{2 d}{d-1}$, we have

$$
\|\widehat{f d \sigma}(\xi)\|_{L^{q}\left(\mathbb{R}^{d}\right)} \leq C\|f\|_{L^{\infty}\left(S^{d-1}, d \sigma\right)},
$$

where $C$ depends only on $d$ and $q$.

This is known for $d=2$ (due to Fefferman and Stein [59]), but remains open for all $d>2$. The range of $q$ is suggested by stationary phase formulas such as (2.11). Plugging in $f \equiv 1$ shows that this range cannot be improved. Indeed, $\widehat{d \sigma}$ can be computed explicitly:

$$
\widehat{d \sigma}(\xi)=2|\xi|^{-\frac{d-1}{2}} \cos \left(2 \pi\left(|\xi|-\frac{d-1}{8}\right)\right)+O\left(|\xi|^{-\frac{d+1}{2}}\right),
$$

which belongs to $L^{q}\left(\mathbb{R}^{d}\right)$ only for $q$ exactly as indicated above.

If instead of assuming that $f \in L^{\infty}$ we make the weaker assumption that $f \in$ $L^{2}\left(S^{d-1}\right)$, then the best possible result is known.

Theorem 2.5. For all $q \geq \frac{2 d+2}{d-1}$, we have

$$
\|\widehat{f d \sigma}(\xi)\|_{L^{q}\left(\mathbb{R}^{d}\right)} \leq C\|f\|_{L^{2}\left(S^{d-1}, d \sigma\right)} .
$$

This was first proved by Stein in 1967 (unpublished) for a smaller range of $q$. In 1975 P.A. Tomas extended the result to $q>\frac{2 d+2}{d-1}$ [189], [190], and later that year the endpoint was settled by Stein [168]. A simple construction known as the Knapp counterexample shows that the range of $q$ in Theorem 2.5 is optimal.

The Tomas-Stein argument is very general and uses only limited information about the geometry of $S^{d-1}$, namely its dimensionality and the decay of $\widehat{\sigma}$ at 

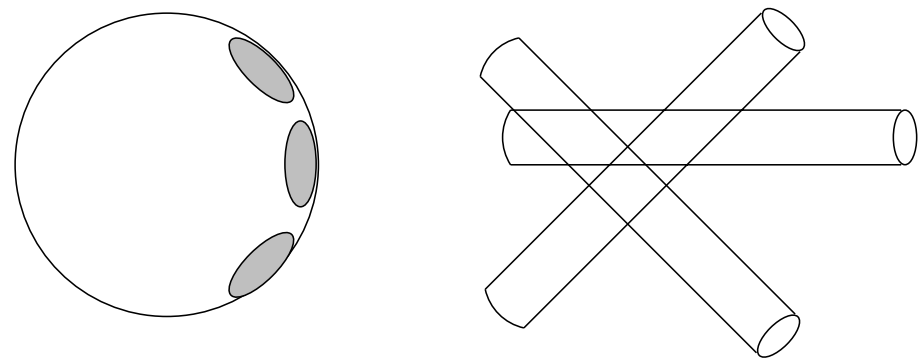

FiguRE 2. Different spherical caps correspond to tubes in different directions in the Fourier space.

infinity. Large parts of the proof have since been adapted to rather different settings, including fractal sets (Mockenhaupt [129]) and sequences of integers (Bourgain [15], Green [79]).

One can interpolate between Tomas-Stein and the trivial $L^{1}-L^{\infty}$ estimate to get a range of intermediate estimates. Going beyond that, however, was much more difficult, and for many years, until Bourgain's breakthrough in 1991 [13], it was not even known whether this was possible at all. It turns out that a substantially new approach was required. While Theorem 2.5 is mostly based on analytic considerations, restriction estimates such as (2.10) with $p>2$ require deeper geometrical information, and this is where we find Kakeya sets lurking under the surface.

Our starting point is that the restriction conjecture (2.12) implies the Kakeya conjecture (Conjecture 1.2). This was first stated and proved formally by Bourgain in [13], but very similar arguments were used in the harmonic analysis literature throughout the 1970s and 1980s (see in particular Córdoba [38]), all inspired by Charles Fefferman's use of Besicovitch sets to produce a counterexample to the closely related ball multiplier conjecture [60]. Below is a rough sketch of this argument, adapted to the restriction setting.

Let $f_{a, \delta}(x)=e^{2 \pi i \eta_{a} x} \chi_{a}(x)$, where $a \in S^{d-1}, \eta_{a} \in \mathbb{R}^{d}$, and $\chi_{a}$ is the characteristic function of the spherical cap centered at $a$ of radius $\delta$ for some very small $\delta>0$. Scaling considerations, standard in harmonic analysis, show that $\overline{f_{a, \delta}}$ is roughly constant on tubes of length $\delta^{-2}$ and radius $\delta^{-1}$. Forgetting about mathematical rigour for a moment, we will in fact think of $\widehat{f_{a, \delta}}$ as the characteristic function of one such tube. Moreover, by adjusting the phase factor $\eta_{a}$ we can place that tube at any desired point in the dual space $\mathbb{R}_{\xi}^{d}$.

Now cover the sphere by finitely overlapping $\delta$-caps, and let $F_{\delta}(x)$ be the sum of the associated functions $f_{a, \delta}$ just defined. Then $\left\|F_{\delta}\right\|_{\infty} \leq C$, uniformly in $\delta$. On the other hand, $\widehat{F_{\delta}}$ is the sum of a large number of characteristic functions of tubes as described above (see Figure 2). If we now arrange these tubes as in the Besicovitch set construction, then the size of the support of $\widehat{F_{\delta}}$ will be very small compared to its $L^{1}$ norm. An application of Hölder's inequality shows that this forces the $L^{q}$ norm of $\widehat{F_{\delta}}$ to blow up as $\delta \rightarrow 0$, contradicting (2.12).

The truly groundbreaking contribution of [13] was the discovery that this reasoning was, to some extent, reversible. More precisely, Bourgain developed an analytic machinery to deduce restriction estimates from Kakeya-type geometric information. It is a difficult and analytically sophisticated argument. Instead of a 
dimension bound for Kakeya sets in $\mathbb{R}^{d}$, we actually need a somewhat stronger result - an $L^{p}$ estimate on the Kakeya maximal function (2.5). This is followed by simultaneous analysis on two different scales (local restriction estimates), combining the maximal function result just mentioned with Tomas-Stein type orthogonality arguments. The numerology produced here is somewhat complicated; in particular there is no natural explanation for the range of the exponents $p$ in Bourgain's theorem.

Further progress on Conjecture 2.4 has followed two lines. One is to improve the known Kakeya maximal function estimates (we will say more about such work in Section 3), then plug them directly into the existing Kakeya-to-restriction conversion machinery, obtaining better restriction exponents as a corollary. The other direction of research is to improve the machinery itself. There is a number of contributions of this type, including work by Moyua-Vargas-Vega [132], Tao-Vargas-Vega [184], Tao-Vargas [185], [186], Tao [176], and Wolff [199]. The updated toolbox includes bilinear restriction estimates, induction on scales, wave packet decompositions, local restriction estimates, and more. A comprehensive review of the modern approach to the subject is given in [177].

The current best result, due to Tao [176], can be explained as follows. Interpolating between the Stein-Tomas theorem (2.13) and the conjectured estimate (2.12) yields a family of conjectured intermediate estimates of the form (2.10). The challenge is to improve the range of $p$ for which such estimates are known. Tao's result is that (2.10) holds with $p>\frac{2(d+2)}{d}$, if $q=q(p)$ is the corresponding exponent from the interpolation. This is obtained as a consequence (via scaling) of a bilinear restriction estimate for paraboloids, proved also in [176] and largely inspired by Wolff's sharp bilinear restriction estimate for the light cone [199].

\section{The Kakeya PRoblem Revisited}

3.1. Geometric arguments. We now return to Conjecture 1.2 and to the quest for bounds on the dimension of Kakeya sets in dimensions $d \geq 3$. Prior to 1991, it was known that the Hausdorff dimension of a Kakeya set in $\mathbb{R}^{d}$ must be at least $(d+1) / 2$; this follows from the x-ray and $k$-plane transform estimates of Drury [43] and Christ [34], although is not stated there explicitly. We begin by sketching an alternative geometric proof due to Bourgain [13], known in the harmonic analysis community as the "bush argument". For simplicity, we will outline only the proofs of upper Minkowski dimension results rather than Hausdorff or maximal function bounds.

Suppose that $E$ is a Kakeya set in $\mathbb{R}^{d}$. For each $e \in S^{d-1}, E$ contains a unit line segment $T^{e}$ in the direction of $e$. Let $\mathcal{E}$ be a maximal $\delta$-separated subset of $S^{d-1}$, so that $|\mathcal{E}| \approx \delta^{-(d-1)}$, and let $T_{\delta}^{e}$ be the $\delta$-neighbourhood of $T^{e}$. Abusing notation only very slightly, we write $E_{\delta}=\bigcup_{e \in \mathcal{E}} T_{\delta}^{e}$. Suppose that $\overline{\operatorname{dim}}_{M}(E)<\alpha$, so that $\left|E_{\delta}\right| \leq C \delta^{d-\alpha}$. Since $\sum_{e \in \mathcal{E}}\left|T_{\delta}^{e}\right| \approx 1$, there must be at least one point, say $x_{0}$, which belongs to at least $\delta^{-(d-\alpha)}$ tubes $T_{\delta}^{e}$. The key observation is that these tubes have at most finite overlap away from a small neighbourhood of $x_{0}$. (Two straight lines can only intersect at one point; see Figure 3). Thus $\left|E_{\delta}\right|$ is bounded from below by a constant times the sum of volumes of the tubes through $x_{0}$ :

$$
\left|E_{\delta}\right| \geq C \delta^{-(d-\alpha)} \cdot \delta^{d-1}=\delta^{\alpha-1} .
$$

But this is only possible if $\alpha-1 \geq d-\alpha$, i.e. $\alpha \geq \frac{d+1}{2}$. 

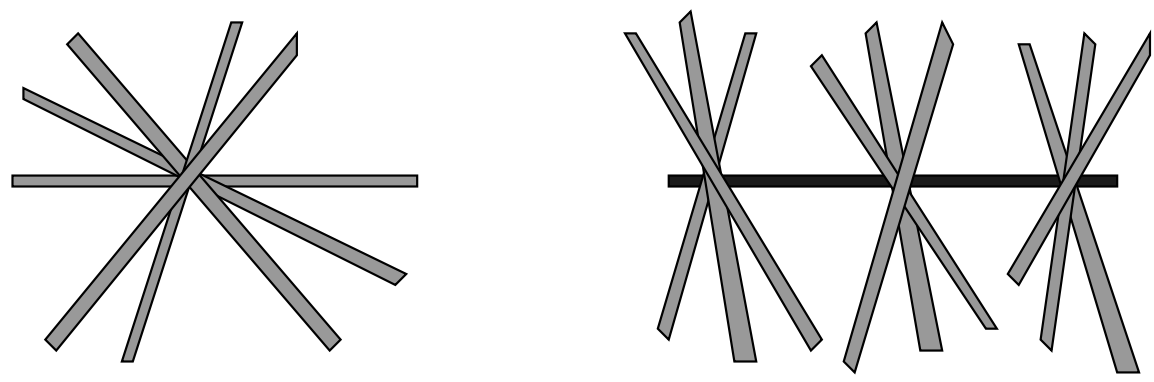

FiguRE 3. A bush and a hairbrush.

In [13], this is supplemented by an additional geometrical argument improving the dimension bound to $\frac{d+1}{2}+\epsilon_{d}$, with $\epsilon_{d}$ given by a recursive formula (for $d=3$ this yields the bound $7 / 3$ ).

A more efficient geometrical argument, leading to the estimate $\operatorname{dim}_{H}(E) \geq \frac{d+2}{2}$, was given a few years later by Tom Wolff [193]. Wolff observes that in order for $E_{\delta}$ to have small volume, it is necessary for a large fraction of the set, not just one point, to have high multiplicity. In fact, many of the tubes $T_{\delta}^{e}$ must consist largely of high multiplicity points. Take one such tube, along with the union of all tubes that intersect it (this object is often called a "hairbrush"). By combining Bourgain's "bush" construction above with an earlier planar estimate due to Córdoba [38], one can prove that the bristles of the hairbrush must be essentially disjoint. We then bound the volume of $E_{\delta}$ from below by the volume of the hairbrush, and the Minkowski dimension estimate again follows upon taking $\delta \rightarrow 0$.

This comes with a few caveats. The hairbrush argument does not quite work as stated and requires some modifications if the tubes of $E_{\delta}$ tend to intersect at very low angles. More importantly, there are additional issues that arise in the calculation of the Hausdorff dimension (as opposed to Minkowski). We will not elaborate on this here, but we do want to mention the two ends reduction of [193], which was introduced to resolve that problem and has inspired some of the induction on scales techniques in restriction theory.

Wolff's argument, although more elaborate than Bourgain's, is still relatively simple in the sense that only very basic geometric information is being used. It was tempting to try to improve on it by using more sophisticated combinatorial methods; thus harmonic analysts were introduced to combinatorial geometry. While the Kakeya problem resisted this approach, many other connections between the two areas were made and explored successfully. We will return to this in Section 4.

3.2. Additive and hybrid arguments. A radically different "arithmetic" approach to the problem was introduced by Bourgain in 1998 [17]. Forget about the hairbrush construction for a moment: we will try to improve on the bush argument instead, in another direction.

Consider a hypothetical Kakeya set $E \subset \mathbb{R}^{d}$ of dimension very close to $(d+1) / 2$. We perform a discretization procedure as in the last subsection, except that we will now ignore the distinction between a tube and a line. We will also restrict our attention to those lines which make an angle less than $\pi / 100$ with the $x_{d}$-axis. Consider the intersections $A, B, C$ of the discretized set $E$ with the three parallel hyperplanes $x_{d}=0, x_{d}=1, x_{d}=1 / 2$, as in Figure 4 (rescale and translate the set 


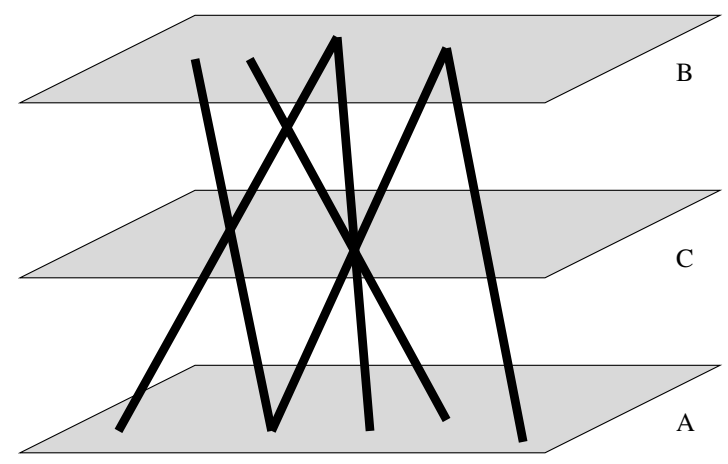

Figure 4. Three slices of a Kakeya set.

if necessary). We consider $A, B, C$ as subsets of $\mathbb{R}^{d-1}$. Let $S=\{(a, b)$ : there is a line from $a$ to $b\}$. Then

$$
\{(a+b) / 2:(a, b) \in S\} \subset C .
$$

The key result is the following lemma.

Lemma 3.1. Let $A, B$ be two subsets of $\mathbb{Z}^{d}$ of cardinality $\leq n$, and let $S \subset A \times B$. If $|\{a+b:(a, b) \in S\}| \leq C n$, then

$$
|\{a-b:(a, b) \in S\}| \leq C^{\prime} n^{2-\frac{1}{13}} .
$$

Assuming Lemma 3.1 for the moment, the proof is completed as follows. Due to multiplicity considerations similar to those in Section 3.1, we have $|A|,|B|,|C| \leq n$ with $n$ close to $\delta^{-(d-1) / 2}$. The lemma then states that $|\{a-b:(a, b) \in S\}| \leq$ $c n^{2-1 / 13}$. But the last set includes the set of "all" directions; hence it must have cardinality about $\delta^{-(d-1)}$, which is greater than the lemma allows if $n$ is too close to $\delta^{-(d-1) / 2}$.

Bourgain worked out a quantitative version of this in [17], obtaining a lower bound $(13 d+12) / 25$ for the dimension of the Kakeya sets in $\mathbb{R}^{d}$, which is better than Wolff's result in high dimensions. The Minkowski dimension argument is more or less as described above, but the Hausdorff and maximal function versions present substantial additional difficulties in arranging a setup where the lemma can be applied.

The bounds in [17] have since been improved in several directions. The arithmetic approach was developed further by Nets Hawk Katz and Terence Tao [112], [114], who first simplified Bourgain's proof of Lemma 3.1 and improved the exponent $1 / 13$ in (3.1) to $1 / 6$, then went on to use four and more "slices". Instead of a single bound on the size of $\{a+b:(a, b) \in S\}$, they assumed bounds on the size of sets of more general linear combinations

$$
\left\{a+\alpha_{i} b: a, b \in S\right\}, i=1, \ldots, k,
$$

where $\alpha_{i}$ are fixed numbers; this allowed them to improve further the exponent in (3.1).

The hybrid arguments of Katz-Laba-Tao [109] and Katz-Tao [114] combine Wolff's geometric combinatorics with Bourgain's arithmetic method. The first hybrid argument, in [109], was originally intended to be geometric. Our goal was to 
improve on Wolff's hairbrush estimate in $\mathbb{R}^{3}$, at least for the Minkowski dimension. Suppose, for the sake of argument, that there were a Kakeya set in $\mathbb{R}^{3}$ of dimension very close to $5 / 2$. Elaborating on the hairbrush estimate (see also Wolff's further arguments in [195]), one can prove that such a set would have to satisfy very strict geometric conditions: for instance it would have to be almost invariant under a rather large family of mappings. Surely this was not possible. Except that we could not pin down the contradiction.

Bourgain's approach, however, provided a way out. Effectively, it said that our hypothetical set would have structure, to the extent that many of its lines would have to be parallel instead of pointing in different directions. Not a Kakeya set, after all.

The current best lower bound on the Hausdorff dimension of Kakeya sets in $\mathbb{R}^{d}$, $d \geq 3$, is

$$
\operatorname{dim}_{H}(E) \geq \max \left(\frac{d+2}{2},(2-\sqrt{2})(d-4)+3\right) .
$$

The first estimate is Wolff's [193]; the second, which is better for $d \geq 5$, is due to Katz and Tao [114]. Further improvements for the Minkowski dimension are in Katz-Łaba-Tao [109], Łaba-Tao [121] and Katz-Tao [114]; and maximal function results are in Wolff [193] and Katz-Tao [114]. A summary of the known bounds is given in the expository paper by Katz and Tao [115]. A theorem of Katz [108] states that the arithmetic approach, as developed in [17], [112], [114], cannot by itself resolve the Kakeya conjecture.

The reader may have forgotten by now that we still have not said anything about the proof of Bourgain's lemma. Lemma 3.1 originates in additive number theory (Section 5); more specifically, it derives from a result known as the Balog-SzemerédiGowers theorem, the relevant version of which was proved by Timothy Gowers in the course of his work on Szemerédi's theorem on arithmetic progressions [73], [74]. The subsequent Katz-Tao improvements rely on additive number theoretic techniques as well.

Bourgain's argument was, to this author's knowledge, the first application of additive number theory to Euclidean harmonic analysis. It was significant, not only because it improved Kakeya bounds, but perhaps even more so because it introduced many harmonic analysts to additive number theory, including Tao, who contributed so much to the subject later on, and jump-started interaction and communication between the two communities. The Green-Tao theorem and many other developments might never have happened were it not for Bourgain's brilliant leap of thought in 1998.

3.3. Extensions and generalizations. A brief mention should be made that many other similar questions have been considered in the literature. A $(d, k)$ set is a set in $\mathbb{R}^{d}$ which contains a translate of each $k$-dimensional subspace. It is known that such sets have positive measure when $k$ is large compared to $d$ (due to Bourgain [13] for $2^{k+1}+k \geq d$, with earlier results due to Marstrand and Falconer). For small $k$ the problem remains open, but partial results are available; see e.g. Oberlin [138]. There is a variety of results on Kakeya and Nikodym sets with a restricted set of directions, e.g. Nagel-Stein-Wainger [133], Katz [107] or Bateman-Katz [3]. Another open question, due to Furstenberg, concerns Kakeyatype sets which, instead of an entire line segment in each direction, contain an $\alpha$-dimensional subset of it for some $0<\alpha \leq 1$ (see Wolff [197] and Katz-Tao [113] 


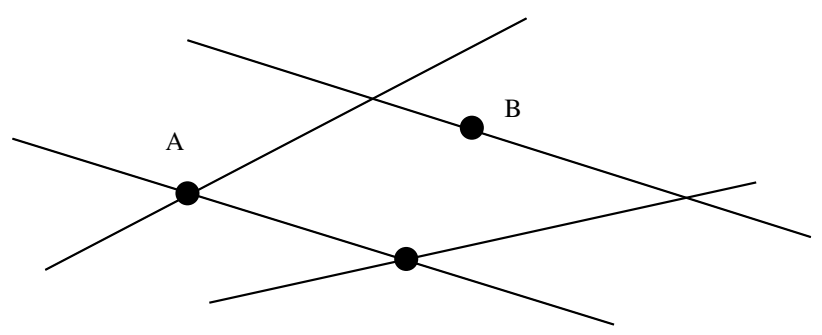

Figure 5. Point-line incidences. There are 2 incidences at $A$ and 1 at $B$.

for a discussion). Similar "packing" questions have been asked for other lowerdimensional geometrical objects, such as circles and spheres (cf. Corollary 2.3 and Section 4.2) or curves with non-vanishing torsion in $\mathbb{R}^{3}$ (see Pramanik-Seeger [145]). Kakeya-type problems on manifolds (Minicozzi-Sogge [128]) and in finite fields (Wolff [197] and Mockenhaupt-Tao [131]) have also been considered. More information on these and other similar questions can be found e.g. in Falconer [58], Stein [169], and Wolff [197].

\section{Combinatorial geometry and harmonic analysis}

4.1. Incidence theorems in combinatorics. Combinatorial geometry studies arrangements of geometric objects (such as lines, planes, circles, convex bodies) in Euclidean spaces. It encompasses a rather wide range of questions, including complexity of geometric arrangements, packing and covering questions, geometric Ramsey theory, and much more. Here we will discuss only one group of problems, all related to counting incidences between points and objects such as lines or surfaces. We will further restrict our attention to a few well-known representative questions; a comprehensive review of this area is in Pach-Sharir [141].

Let $P$ be a set of points and $\mathcal{S}$ a family of lines in a 2-dimensional plane. An incidence is a pair $(x, \ell)$ such that $x \in P, \ell \in \mathcal{S}$, and $x \in \ell$; we will also say that $\ell$ is incident to $x$ if $x \in \ell$ (see Figure 5). Incidences between points and curves, or points and surfaces in higher dimensions, are defined similarly. Paul Erdős, who could see patterns and bounds in seemingly chaotic environments, conjectured in 1946 that there should be a non-trivial upper bound on the number of incidences between $n$ points and $m$ lines in the plane and that a lattice-like arrangement would be optimal in that respect if $n \approx m$. This was indeed proved by Szemerédi and Trotter in 1983 [175].

Theorem 4.1. The number of incidences between $n$ lines and $m$ points in $\mathbb{R}^{2}$ is at most

$$
O\left(m+n+m^{2 / 3} n^{2 / 3}\right)
$$

and this estimate is sharp.

Two alternative proofs of Theorem 4.1 were quite influential in their own right. The cell decomposition technique of Clarkson et al. [37] has quickly found many other applications, for example to the incidence problems for circles which we will discuss shortly. Székely's surprisingly short and elegant proof [171], based on the crossing lemma in graph theory, had the added advantage of allowing the rather 
astronomical implicit constant in the original Szemerédi-Trotter proof to be reduced to a more manageable 2.57 (see the discussion in Pach-Sharir [141]). Note that the "important" term in (4.1) is $m^{2 / 3} n^{2 / 3}$; the $m$ and $n$ terms dominate only in certain degenerate cases.

Consider now a similar question for circles in $\mathbb{R}^{2}$ : how many incidences can there be between $m$ circles and $n$ points in the plane? The best current bound in the case $m \approx n$ is $O\left(n^{1.364}\right)$, due to Aronov and Sharir [1]; for general $n$ and $m$, the estimate is more complicated and distinguishes between several cases. This bound is weaker than (4.1) - as is to be expected, given that circles have more "degrees of freedom" than lines. A straight line is determined uniquely by 2 points; the same is not true for circles.

Circles are, however, determined by 3 points. Is this a sufficient basis for a nontrivial incidence bound? Pach and Sharir [140] have indeed proved an incidence theorem for pseudolines, planar curves determined uniquely by $k$ points, with exponents depending on $k$. The Aronov-Sharir bound cited above is slightly better than the $k=3$ case of [140]: it uses additional geometrical information about circles which is not available for more general pseudolines.

Incidence theorems in higher dimensions can be rather more troublesome. It is not even always clear how to formulate the question. Consider, for example, $n$ points and $m$ planes in $\mathbb{R}^{3}$ : if all points lie on a fixed line $\ell$ and all planes pass through $\ell$, then every point is incident to every plane. It is reasonable to expect, though, that there should be non-trivial incidence bounds for "non-degenerate" arrangements in which such lower-dimensional obstructions do not occur. Formulating appropriate non-degeneracy conditions then becomes part of the problem. The "right" bound on the number of plane-point incidences in $\mathbb{R}^{3}$ appears to be $O\left(m^{3 / 4} n^{3 / 4}\right)$ (see e.g. Elekes-Tóth [49], Laba-Solymosi [120], and Solymosi-Tóth [163]), but the exact statement of the result depends on what additional assumptions are made. We omit the fine print.

In a 3-page paper published in 1946 [52], Erdős formulated several elementarylooking questions on sets of distances between $n$ points. These questions keep the combinatorial geometers busy to this day. One is the unit distances problem: given a set $E$ of $n$ points in $\mathbb{R}^{d}$, how many pairs $x, y \in E$ can there be with $|x-y|=1$ ? This can be reformulated as an incidence problem for unit spheres, as we have $|x-y|=1$ if and only if $x$ is incident to the unit sphere centered at $y$. In $\mathbb{R}^{2}$, a variant of the Szemerédi-Trotter theorem shows that the number of such incidences is bounded by $O\left(n^{4 / 3}\right)$ (Spencer-Szemerédi-Trotter [166]). This is the best result to date, but "it is likely" [52] that the optimal bound should be $O\left(n^{1+\epsilon}\right)$ for any $\epsilon>0$.

In three dimensions, the number of unit distances is known to be bounded by $O\left(N^{\frac{3}{2}+\epsilon}\right)$ for any $\epsilon>0$ (Clarkson et al. [37]), and one cannot do better than $O\left(n^{4 / 3} \log \log n\right)$ (attributed to Erdős in [141]). However, well-known examples show that there can be no such non-trivial bounds in dimensions $d \geq 4$. For instance, a set of $2 n$ points in $\mathbb{R}^{4}, n$ of which lie on the circle $x_{1}^{2}+x_{2}^{2}=\frac{1}{2}$ in the $\left(x_{1}, x_{2}\right)$ coordinate plane, and the other $n$ on a similar circle in the $\left(x_{3}, x_{4}\right)$ plane, has $n^{2}$ unit distances.

Define the distance set of $E$ to be

$$
\Delta(E)=\{|x-y|: x, y \in E\}
$$


How small can $|\Delta(E)|$ be if $|E|=n$ ? This is the distinct distances problem, also first posed by Erdős in [52]. Lattice-like examples in [52] show that $|\Delta(E)|$ can be as small as $n / \sqrt{\log n}$ for $d=2$ and $n^{2 / d}$ in higher dimensions, which is widely believed to be optimal.

It is possible to reformulate the question in terms of incidences; indeed, an optimal bound $n^{1+\epsilon}$ for the unit distance problem in the plane would also resolve the 2-dimensional distinct distances problem. A straightforward approach along these lines, however, would require much stronger incidence theorems than those currently available. The best strategy to date, due to Solymosi and Tóth [162], combines in an ingenious way the Szemerédi-Trotter theorem, the crossing lemma mentioned earlier, a related theorem of Beck, and a combinatorial number theoretic argument. By improving the latter, Katz and Tardos [116] have obtained the current best lower bound $|\Delta(E)| \geq C n^{0.8641 \ldots}$ for $d=2$.

In higher dimensions, the number of distinct distances is known to be at least $n^{2 / d-1 / d^{2}}$ for all $d$ and $n^{.5794}$ for $d=3$ (Solymosi-Vu [164]). We also mention that earlier results for $d=2$ were due to Erdős, Moser, Chung, Chung-SzemerédiTrotter, and Székely.

4.2. What harmonic analysts have learned. Combinatorial geometry is nowadays a standard tool for many harmonic analysts. The Szemerédi-Trotter theorem, incidence theorems, distance set bounds have become household words in the harmonic analysis literature. Communication and collaboration between the two areas is becoming more frequent.

It is easy to forget that this was not always the case. Not that harmonic analysts were unaware of the geometric content of their work - quite the opposite. As late as the early 1990s, however, harmonic analysis and combinatorial geometry existed in parallel planes, mostly unaware of each other. It did not help that combinatorial and computational geometers were often on the faculty of computer science, rather than mathematics, departments. References to combinatorial-geometric literature are very hard to find in pre-1995 work in harmonic analysis.

The shift was largely due to Tom Wolff's pioneering work in the 1990s. Wolff introduced harmonic analysts to incidence geometry, popularizing it in his lectures and incorporating combinatorial-geometric methods in his own work. His attempts to approach the Kakeya problem via techniques from incidence geometry, such as higher-dimensional variants of the Szemerédi-Trotter theorem, ultimately failed to improve the known dimension bounds. (One such effort is described in more detail in [197].) However, Wolff was much more successful with other questions. One of his greatest achievements was his work in [198] on the local smoothing conjecture, formulated by Sogge in [158].

Conjecture 4.2. If $u(x, t)$ solves the Cauchy problem for the wave equation in $\mathbb{R}^{d+1}$ :

$$
u_{t t}=\Delta_{x} u, u(x, 0)=f(x), u_{t}(x, 0)=g(x),
$$

then there is a space-time regularity estimate

$$
\|u\|_{L^{p}\left(\mathbb{R}^{d} \times[1,2]\right)} \leq C\left(\|f\|_{p, \alpha}+\|g\|_{p, \alpha-1}\right), \alpha>\frac{1}{2}-\frac{2}{p},
$$

for $p \geq \frac{2 d+2}{d-1}$, where $\|\cdot\|_{p, \alpha}$ is the inhomogeneous $L^{p}$ Sobolev norm with $\alpha$ derivatives. 
Conjecture 4.2, while somewhat related to the Strichartz estimates mentioned briefly in Section 2, turns out to be much deeper and more difficult. It follows from results of Sogge and Tao that Conjecture 4.2 formally implies the restriction and Kakeya conjectures (see [177] and [197] for a discussion).

Wolff proved in [198] that (4.2) holds for $d=2$ and $p>74$. Although weaker estimates had been available earlier, this was the first time that (4.2) was proved for any finite $p$ with the sharp range of $\alpha$, which was a critical difficulty. Wolff's result was later extended to $d \geq 3$ by Laba-Wolff [122], and the range of $p$ has been improved by Garrigós-Seeger [70]; see also Pramanik-Seeger [145] for further extensions.

Wolff's proof was a tour de force, both analytically and combinatorially. The analytic methods were in the spirit of those described in Section 2 in connection with the restriction conjecture; in particular the wave packet decomposition and induction of scales played a major role. The combinatorial part involved incidences for circles. The main geometric result of [198] is somewhat complicated to state, but the following question, addressed explicitly in Wolff's paper [194] on BRK sets, is very closely related to it.

Given $n$ circles in $\mathbb{R}^{2}$, no three tangent at a point, how many pairs of tangent circles can there be?

A BRK, or Besicovitch-Rado-Kinney, set is a Borel set in $\mathbb{R}^{d}, d \geq 2$, which contains a sphere of every radius. It is known (due independently to BesicovitchRado and Kinney) that there are BRK sets of $d$-dimensional measure 0. Kolasa and Wolff [117] and Wolff [194] proved that BRK sets in $\mathbb{R}^{d}$ must have Hausdorff dimension $d$. The first paper [117], published in 1999 but first circulated in 1994, settled the easier case $d \geq 3$ and gave a partial result for $d=2$. The remaining obstacle in 2 dimensions was the tangency question stated above.

In [194], Wolff resolved the 2-dimensional case by applying the combinatorial cell decomposition technique of Clarkson et al. [37], which he learned only after [117] was completed, to the tangency question. His bound $O\left(n^{3 / 2+\epsilon}\right)$ on the number of tangencies, while probably not optimal, was nonetheless sufficient to settle the BRK problem. A more refined version of that argument is essential in [198], where Wolff also states the following geometric corollary.

Let $F \subset \mathbb{R}^{2}$ be a compact set. If there is a compact set $E \subset$ $\mathbb{R}^{2}$ with Hausdorff dimension greater than 1 such that $F$ contains some circle centered at each point of E, then $F$ has positive 2dimensional measure.

Changing tacks, we turn to distance sets. The following conjecture, formally an analogue of the combinatorial distinct distances problem stated in Section 4.1, is due to Falconer [57].

Conjecture 4.3. Let $E$ be a compact set in $\mathbb{R}^{d}, d \geq 2$. If $E$ has Hausdorff dimension $\operatorname{dim}_{H}(E)>d / 2$, then its distance set

$$
\Delta(E)=\{|x-y|: x, y \in E\}
$$

has positive 1-dimensional Lebesgue measure.

The current best result in this direction is that

$$
|\Delta(E)|>0 \text { if } \operatorname{dim}_{H}(E)>\frac{d}{2}+\frac{1}{3},
$$


due to Wolff [196] for $d=2$ and Erdog̃an [50], [51] for $d \geq 3$.

Both Wolff and Erdog̃an employ a Fourier-analytic strategy, originally due to Mattila [126] (although similar arguments had been used by Kaufmann in a somewhat different context) and used also by Bourgain in [16]. The key ingredient is the decay of the spherical averages of $\widehat{\mu}$, where $\mu$ is an appropriate measure supported on $E$. Such estimates are closely related to restriction estimates: Wolff's proof is reminiscent of his work on the Kakeya and restriction problems, while Erdogan relies directly on Tao's paraboloid restriction estimate [176]. As in restriction problems, both dimensionality (via energy integrals of $\mu$ ) and curvature (of the sphere, the level set of the distance function) are crucially involved.

Can we apply discrete-geometric methods, for example those of Solymosi-Tóth [162] and Katz-Tardos [116], to the continous conjecture?

No, not in this case. True, there are discretization procedures that can match a continuous problem to its discrete analogue. They are used, for instance, in [194], [198], and in many papers on the Kakeya problem. Here, however, the equivalence simply cannot be made rigorous.

On the other hand, harmonic analytic techniques yield certain results not available by combinatorial methods. Consider, for example, distance sets defined with respect to a non-Euclidean distance function in $\mathbb{R}^{d}, d \geq 3$, with a convex hypersurface $\Gamma$ as the unit sphere. Combinatorial techniques seem to be of little use in this setting, mainly because intersections of convex bodies in dimensions $d \geq 3$ are very difficult to control. However, if $\Gamma$ is smooth and has non-vanishing Gaussian curvature, we still have the continuous estimate (4.3), by Erdogan's argument with only minor modifications. A continuous-to-discrete conversion mechanism, which turns out to work in this direction, yields a non-trivial discrete bound (IosevichHofmann [101], Iosevich-Łaba [104]). For more details and further results of this type, see Iosevich-Hofmann [101], Iosevich-Łaba [104], and Kolountzakis [118].

Very recently, in Iosevich-Jorati-Łaba [102] we used Sobolev regularity estimates for averaging operators of the form

$$
T f(x)=\int_{\Gamma_{x}} f(y) d \sigma_{x}(y),
$$

where $\left\{\Gamma_{x}\right\}$ is a family of hypersurfaces equipped with surface measures $\sigma_{x}$, to prove discrete incidence theorems. This yields surprisingly good results: while we cannot quite match, for instance, the known combinatorial bounds for unit spheres, our results extend naturally to more general cases, such as spheres with slowly varying radii and other smooth curved hypersurfaces.

Many other connections between harmonic analysis and geometric combinatorics have been explored in the literature; see e.g. Carbery-Christ-Wright [29], Christ [35], Iosevich-Katz-Tao [103], and Schlag [154], [155] for a few well-known examples.

\section{AdDitive Number THEORY}

5.1. Freiman's theorem. Additive number theory is sometimes thought to straddle the gap between number theory and combinatorics. It studies additive properties of sets of numbers, drawing on a wide variety of methods from number theory, combinatorics, and discrete harmonic analysis. Its questions and theorems are often stated in the language of grade-school arithmetic - addition, multiplication, counting of integers - but this can belie the complexity of the arguments involved. Once more, we will focus on a small number of well-known problems representative 
of the area; for more comprehensive surveys see e.g. Croot-Lev [39], Granville [78], Nathanson [135], and Tao-Vu [187]. We begin with Freiman's theorem, a fundamental result on set addition.

Let $A \subset \mathbb{Z}$ be a finite set, and let $A+A=\{a+b: a, b \in A\}$. It is easy to prove that $|A+A| \geq 2|A|-1$ and that the equality holds if and only if $A$ is an arithmetic progression. But what if we know only that $|A+A| \leq K|A|$ for some (possibly large) constant $K$ ? Does this imply that $A$ has arithmetic structure? Of course $A$ could be an arithmetic progression again, or a large subset thereof, but it could also have the more general lattice-like form

$$
A=\left\{a+j_{1} r_{1}+\cdots+j_{m} r_{m}: 0 \leq j_{i} \leq J_{i}, i=1, \ldots, m\right\},
$$

with $m$ small enough depending on $K$. Such sets are called generalized arithmetic progressions of dimension $m$. Freiman's theorem [63], [64] asserts that all sets with small sumsets are large subsets of generalized arithmetic progressions.

Theorem 5.1. Suppose that $A \subset \mathbb{Z}$ and that $|A+A| \leq K|A|$. Then $A$ is contained in a generalized arithmetic progression (5.1) of size at most $C|A|$ and dimension at most $m$, where $C$ and $m$ depend only on $K$.

Freiman's original proof was rather difficult to follow and the community owes a great debt to Imre Ruzsa, who essentially rediscovered Theorem 5.1, gave it its present formulation and provided a much more accessible proof [152]. For these reasons, Theorem 5.1 is sometimes referred to as the Freiman-Ruzsa theorem.

Ruzsa's proof of Theorem 5.1 combined ingenious combinatorial arguments, discrete Fourier analysis, Minkowski's second theorem in the geometry of numbers, and a formerly obscure result in graph theory (Plünnecke inequalities), which Ruzsa also rediscovered and made accessible. Bilu [10] subsequently reworked and clarified Freiman's original geometric ideas. The current best quantitative bounds are due to Mei-Chu Chang [30], who followed the general outline of Ruzsa's argument but with several improvements, one of which (a theorem on the structure of the set of large Fourier coefficients of a function) has found a variety of other applications. An excellent exposition of the Ruzsa-Chang proof is in [80].

Freiman's theorem has been extended to other abelian groups - the most general result of this type was recently obtained by Green and Ruzsa [84].

Theorem 5.1 is notable for the simplicity and elegance of its statement, but applications often call for other variants. For example, it is sometimes necessary to apply a Freiman-type result in a setting where, instead of a bound on the size of the entire sumset $A+A$, we know only that $A$ has weaker additive properties. One such case is addressed by the Balog-Szemerédi-Gowers theorem.

Theorem 5.2. Let $A \subset \mathbb{Z}^{d}$. Suppose that the equation $a_{1}+a_{2}=a_{3}+a_{4}$ has at least $\alpha|A|^{3}$ solutions $a_{1}, a_{2}, a_{3}, a_{4} \in A$. Then there is a subset $A^{\prime} \subset A$ such that $\left|A^{\prime}\right| \geq c|A|$ and $\left|A^{\prime}+A^{\prime}\right| \leq C\left|A^{\prime}\right|$, where $c, C$ depend only on $\alpha$.

In particular, it follows from Theorem 5.1 that $A$ has a large intersection with a generalized arithmetic progression. Simple examples show that one cannot say much about all of $A$ under the assumptions of Theorem 5.2: consider a union of two sets of equal cardinality, one of which is an arithmetic progression and the other is entirely random. 
Theorem 5.2 was originally proved by Balog and Szemerédi in [2]. As it turned out, Gowers [73], [74] needed a similar statement in his work on Szemerédi's theorem, but with much stronger quantitative bounds than could be extracted from [2]. He went on to find a new simpler proof of the theorem which also yielded polynomial bounds on $c, C$ in terms of $\alpha$. It was this result that inspired Bourgain's work [17] described here in Section 3.2; his Lemma 3.1 is a modification of Gowers's version of Theorem 5.2.

There is a well-known open problem regarding the size of the "doubling constants" in Freiman's theorem. According to Chang's version of Theorem 5.1 ([30], Theorem 2), a set $A$ with $|A+A| \leq K|A|$ is contained in a generalized arithmetic progression $P$ of dimension $m \leq\lfloor K-1\rfloor$. Explicit examples show that the dimension bound cannot be better than a linear function of the doubling constant $K$. However, $P$ itself will usually have a doubling constant of the order $2^{m}$, which is much larger than $K$. It is widely believed that $A$ must in fact have more structure, for example that it can be covered by a small number of generalized arithmetic progressions of dimension about $\log K$. That has not been proved, though, and it is not even clear how exactly to formulate the conjecture. See e.g. Gowers [75], Green [81], and Green-Tao [88] for further discussion.

5.2. The sum-product problem. Let $A$ be a set of integers. We continue to write $A+A=\left\{a+a^{\prime}: a, a^{\prime} \in A\right\}$; let also $A \cdot A=\left\{a a^{\prime}: a, a^{\prime} \in A\right\}$. The following conjecture was made by Erdős and Szemerédi in [53].

Conjecture 5.3. For any $\epsilon>0$, we have

$$
\max (|A+A|,|A \cdot A|) \geq c_{\epsilon}|A|^{2-\epsilon} .
$$

It is not difficult to arrange for one of $A+A$ and $A \cdot A$ to be small. Just let $A$ be an arithmetic progression, and $A+A$ will have size comparable to that of $A$. Then, however, $A \cdot A$ is large. The situation is reversed if $A$ is a geometric progression: $A \cdot A$ is small, but $|A+A| \approx|A|^{2}$. Additive structure is not compatible with multiplicative structure.

The main challenge is to bridge the gap between the two extreme cases. Structure theorems can be applied at the endpoints [31], [32], but they can only go so far: the conclusion of Freiman's theorem becomes trivial as soon as $|A+A| \approx|A|^{1+\epsilon}$. Conjecture 5.3 asserts that even very weak partial additive and multiplicative structures are mutually exclusive.

Surprisingly, after the first number-theoretic partial results of Erdős-Szemerédi, Nathanson and Ford, a major breakthrough came from incidence geometry. In 1997 Elekes [46] deduced the bound

$$
\max (|A+A|,|A \cdot A|) \geq c|A|^{5 / 4}
$$

from the Szemerédi-Trotter theorem. A more elaborate argument due to Solymosi [160] improves the right side of $(5.3)$ to $|A|^{14 / 11}(\log |A|)^{-3 / 11}$, which is the best result to date.

Elekes's proof is short enough to be given here in full. Let $|A|=n$, and consider the family of $n^{2}$ lines $y=a(x-b), a, b \in A$, in the $x y$-plane. Each line is incident to the $n$ points $(b+c, a c), c \in A$; thus there are at least $n^{3}$ incidences between the $n^{2}$ lines and the point set $P=(A+A) \times(A \cdot A)$. By Theorem 4.1, we have

$$
n^{3} \leq C\left(n^{2}\right)^{2 / 3}|P|^{2 / 3}
$$


hence $|P| \geq C n^{5 / 2}$ and (5.3) follows.

Among other partial results, we mention that both endpoints have been settled: if $|A+A| \leq C|A|$, then $|A \cdot A| \geq|A|^{2} / \log |A|$ (Chang [31], Elekes-Ruzsa [48], and Solymosi [160]), and conversely, $|A \cdot A| \leq C|A|$ implies that $|A+A| \geq c|A|^{2}$ (Chang $[32])$. There are many extensions and generalizations of Conjecture 5.3, including iterated sums and products, sumsets and product sets of distinct sets, sums and products along graphs (Balog-Szemerédi-style), and sums and products in rings other than $\mathbb{Z}$ (real numbers, complex numbers, matrices). See e.g. Bourgain-Chang [24], Chang [31], [32], Elekes [47], and Solymosi [161] for a few examples, and Chang [33] for a brief summary of the known results.

One such extension is currently stealing the show. Far from being a novelty result, it has already found a wide variety of applications, from Mordell type exponential sums in analytic number theory (Bourgain-Glibichuk-Konyagin [26], Bourgain [20], [21]) to explicit constructions of expanders (Bourgain-Gamburd-Sarnak [25]); see Bourgain [22] for a more detailed exposition.

Theorem 5.4. [27], [26] Let $\mathbb{F}_{p}$ be the finite field of integers modulo $p$, where $p$ is a large prime. Then for all $0<\epsilon<1$ there is a $\delta>0$ such that if $A \subset \mathbb{F}_{p}$ and $|A|<p^{1-\epsilon}$, then

$$
\max (|A+A|,|A \cdot A|) \geq c|A|^{1+\delta} .
$$

Theorem 5.4 was first proved by Bourgain, Katz and Tao [27] with the additional assumption that $|A|>p^{\epsilon}$; the latter condition was removed by Bourgain, Glibichuk and Konyagin in [26]. Further improvements are in Iosevich-Hart-Solymosi [100], Garaev [69], and Katz-Shen [110], [111].

We emphasize here that the incidence-based approach, so effective for real numbers, does not quite work the same way in finite fields. Prior to [27], all proofs of the Szemerédi-Trotter theorem and its extensions relied on topological properties of the underlying space (for instance, a straight line cuts a plane in two parts) which have no useful analogue in finite fields. A Szemerédi-Trotter-type theorem in finite fields is indeed proved in [27], but only as a consequence of the sum-product estimate. More recently, Iosevich-Hart-Solymosi [100] gave a new proof of Theorem 5.4 based on incidence theorems for hyperbolas in finite fields; the latter are deduced from number-theoretic estimates on Kloosterman exponential sums.

The Bourgain-Katz-Tao theorem can instead be traced back to, of all things, an unsuccessful 1998 attempt to improve Kakeya dimension bounds in $\mathbb{R}^{3}$. Katz and Tao had tried to argue that a Kakeya set of dimension $5 / 2$ in $\mathbb{R}^{3}$ could be parametrized by an approximate $1 / 2$-dimensional subring of $\mathbb{R}[113]$. This led them to an old question of Erdős and Volkmann [55]:

\section{If $E \subset \mathbb{R}$ is a Borel set and a subring of $\mathbb{R}$, must it have Hausdorff dimension either 0 or 1 ?}

Falconer [56] had proved that there are no Borel subrings with $1 / 2<\operatorname{dim}_{H}(E)<1$; Katz and Tao needed to go just past the $1 / 2$ threshold. In the end, their argument could not be completed, and the 5/2 Kakeya bound was improved in [109] by other means.

A few years later, however, Bourgain resolved the Erdős-Volkmann question in the affirmative [19] - only to find out that G.A. Edgar and C. Miller had just published their own short and elegant solution in [44]. Bourgain's proof, clearly influenced by the Katz-Tao paper [113], was based on more complicated arguments 
from additive number theory. The Bourgain-Katz-Tao article [27] combined the arguments of all three of [19], [44], and [113]. Among the results of [27] the reader will find the 3-dimensional Kakeya argument, once abandoned by Katz and Tao, now made rigorous in the finite field setting.

5.3. Szemerédi's theorem. We will say that a set $A \subset \mathbb{N}$ has upper density $\delta$ if

$$
\varlimsup_{N \rightarrow \infty} \frac{|A \cap[1, N]|}{N}=\delta .
$$

Motivated by van der Waerden's theorem in Ramsey theory, Erdős and Turán [54] conjectured in 1936 that any set of integers $A$ of positive upper density must contain arithmetic progressions of any finite length. This was indeed proved by Klaus Roth [149] for 3-term progressions (with quantitative improvements due to Szemerédi [174], Heath-Brown [95] and Bourgain [18], [23]), then by Szemerédi [172], [173] for progressions of any length. Below is an equivalent statement of this result.

Theorem 5.5. For any $\delta>0$ and any integer $k$ there is an $N(\delta, k)$ such that if $N>N(\delta, k)$ and $A$ is a subset of $\{1,2, \ldots, N\}$ of cardinality $|A| \geq \delta N$, then $A$ must contain a non-trivial $k$-term arithmetic progression.

As of now, Szemerédi's theorem has four remarkably distinct proofs, each of which was a milestone in combinatorics in its own right. The original combinatorial proof by Szemerédi [173], ingenious and complicated even by Szemerédi's standards, featured the regularity lemma, which has since become an invaluable tool in several areas of combinatorics. Furstenberg's ergodic-theoretic proof [66], based on his multiple recurrence theorem, has led to a variety of extensions and generalizations, such as the multidimensional Szemerédi theorem due to Furstenberg and Katznelson [67] and the polynomial Szemerédi theorem of Bergelson and Leibman [9]. Gowers's proof [73], [74] is often referred to as "harmonic analytic", for its structural resemblance to Roth's proof for $k=3$ as well as its actual use of harmonic analysis. It yields an explicit quantitative bound on $N(\delta, k)$ for $k \geq 4$,

$$
N(\delta, k) \leq 2^{2^{\delta^{-2^{k+9}}}}
$$

which was recently improved by Green and Tao [91] for $k=4$ but remains the best available for $k \geq 5$. Finally, there is a more recent hypergraph proof, due independently to Gowers [76], [77] and Nagle-Rödl-Schacht-Skokan [134], [146], [147], [148].

Theorem 5.5 may have been proved several times over, but nonetheless it remains at the center of considerable research activity that shows no signs of slowing down. In ergodic theory, there is a number of recent results on the convergence of multiple ergodic averages in Furstenberg's multiple recurrence theorem and its extensions, including Host-Kra [98], Ziegler [201], Bergelson-Host-Kra [8], Frantzikinakis-Kra [62], Leibman [123], and Tao [183]. Another direction of research is to find quantitative proofs of results, such as the multidimensional Szemerédi theorem, previously available only by ergodic (infinitary) methods; see e.g. Solymosi [159], Gowers [76], [77], or Shkredov [156]. The hypergraph approach continues to be developed; see e.g. Tao [179] and Elek-Szegedy [45].

All known proofs of Szemerédi's theorem rely on a certain dichotomy between randomness and structure. Roughly speaking, if the elements of $A$ were chosen from $\{1, \ldots, N\}$ independently at random, each with probability $\delta$, then with high 
probability there would be about $\delta^{k} N^{2} k$-term arithmetic progressions in $A$, as there are about $N^{2} k$-term arithmetic progressions in $\{1, \ldots, N\}$, each of which would be contained in $A$ with probability $\delta^{k}$. The same is true if $A$ imitates a random set closely enough, in a sense that needs to be made precise. On the other hand, a non-random set should have a certain amount of additive structure, reminiscent of that in Freiman's theorem but much weaker. We use this structure to our advantage, for example by passing to a long arithmetic subprogression of $\{1, \ldots, N\}$ on which $A$ has higher density and then iterating the argument. The challenge is to find a notion of randomness which is strong enough to guarantee existence of $k$-term arithmetic progressions but also weak enough so that its failure implies useful structural properties.

We illustrate this by taking a brief look at Roth's proof for $k=3$. We will identify $\{1, \ldots, N\}$ with the additive group $\mathbb{Z}_{N}$, and let $A \subset \mathbb{Z}_{N},|A|=\delta N$. Given functions $f_{1}, f_{2}, f_{3}: \mathbb{Z}_{N} \rightarrow \mathbb{C}$, define

$$
\Lambda_{3}\left(f_{1}, f_{2}, f_{3}\right)=N^{-2} \sum_{x, r} f_{1}(x) f_{2}(x+r) f_{3}(x+2 r) .
$$

If $A(x)$ is the characteristic function of $A, \Lambda_{3}(A, A, A)$ equals $N^{-2}$ times the number of 3 -term arithmetic progressions in $A$, including the trivial ones with $r=0$. We are ignoring a minor technical issue here, namely the distinction between genuine arithmetic progressions and arithmetic progressions modulo $N$ in $\mathbb{Z}_{N}$; this is easy to fix, for example by assuming that $A \subset\{0, \ldots,\lfloor N / 3\rfloor\}$.

The expression (5.5) turns out to be tailor-made for Fourier analysis. We define the discrete Fourier transform on $\mathbb{Z}_{N}$ by

$$
\widehat{f}(\xi)=N^{-1} \sum_{x=1}^{N} f(x) e^{-2 \pi i x \xi / N} .
$$

A short calculation shows that

$$
\begin{aligned}
\Lambda_{3}(A, A, A) & =\sum_{\xi} \widehat{A}(\xi)^{2} \widehat{A}(-2 \xi) \\
& =\widehat{A}(0)^{3}+\sum_{\xi \neq 0} \widehat{A}(\xi)^{2} \widehat{A}(-2 \xi)=: I_{1}+I_{2},
\end{aligned}
$$

where $I_{1}$ and $I_{2}$ will be interpreted as the main term and the error term, respectively. We trivially have $I_{1}=\delta^{3}$, and if there were no $I_{2}$ term, (5.6) would yield $\delta^{3} N^{2}$ arithmetic progressions in $A$, exactly the number suggested by the random model. In practice, of course, we cannot count on $I_{2}$ to actually vanish. There is a useful substitute, though. We will say that $A$ is 2 -uniform if

$$
|\widehat{A}(\xi)| \leq \delta^{2} / 2 \text { for all } \xi \neq 0 .
$$

It follows immediately from (5.7) and Parseval's identity that $\left|I_{2}\right| \leq \delta^{3} / 2$; hence $\Lambda_{3}(A, A, A) \geq \delta^{3} / 2$. If $N$ is large enough, this means that $A$ must contain nontrivial 3 -term arithmetic progressions. We will refer to the case when (5.7) holds as the "random" case.

It remains to consider the "structured" case when (5.7) fails: there is a $\xi \neq 0$ such that

$$
\frac{\delta^{2}}{2} \leq|\widehat{A}(\xi)|=\left|N^{-1} \sum_{x} A(x) e^{-2 \pi i \xi x / N}\right| .
$$


The last expression, sans the absolute value, can be interpreted as the $L^{2}$ inner product of $A(x)$ and $e^{2 \pi i \xi x / N}$. We say that $A(x)$ has "linear bias" in the sense that it correlates with the periodic function $e^{2 \pi i \phi(x)}$, where $\phi(x)=\xi x / N$ is linear. In the terminology popularized later by Green and Tao (see e.g. [87]), we have unknowingly proved an inverse theorem for the Gowers $U^{2}$ norm. A reasonably short argument based on the periodicity of $e^{2 \pi i \phi(x)}$ shows now that $A$ is biased along certain long arithmetic progressions; in particular there is an arithmetic progression $P$ of length about $\delta^{2} \sqrt{N}$ on which $A$ has density at least $\delta+\delta^{2} / 100$.

The proof of Roth's theorem is completed by iterating the argument. In the random case, we are done. In the structured case, we replace $\{1, \ldots, N\}$ by $P$ and $A$ by $A \cap P$, then start over again by testing for 2-uniformity. If at any time we find ourselves in the random case, the proof is complete. Otherwise, the density must increase by at least $\delta^{2} / 100$ at each step, eventually reaching 1 , at which point the conclusion of the theorem becomes trivial.

Gowers's proof for arbitrary $k$ - the only one that we will discuss here in some detail - follows the same general scheme. However, when $k \geq 4$, the 2-uniformity condition (5.7) can no longer guarantee an abundance of $k$-term progressions. The new key concept is higher-order uniformity, expressed in terms of the Gowers $U^{d}$ norms. The $U^{2}$ Gowers norm is

$$
\|f\|_{U^{2}}=\left(N^{-3} \sum_{x, r_{1}, r_{2}} f(x) \overline{f\left(x+r_{1}\right) f\left(x+r_{2}\right)} f\left(x+r_{1}+r_{2}\right)\right)^{1 / 4}
$$

the general definition is similar, but the averages are taken over $d$-dimensional "boxes" $\left\{x+\omega_{1} r_{1}+\cdots+\omega_{d} r_{d}: \omega_{1}, \ldots, \omega_{d} \in\{0,1\}\right\}$.

Let $A \subset \mathbb{Z}_{N},|A|=\delta N$, and let $f^{A}(x)=A(x)-\delta$. We call $A d$-uniform if

$$
\left\|f^{A}\right\|_{U^{d}} \leq c(\delta)
$$

with $c(\delta)$ sufficiently small. A short calculation shows that this is equivalent to (5.7) when $d=2$ (modulo the choice of $c(\delta)$ ), but for $d \geq 3$ it is a strictly stronger condition.

If (5.10) holds with $d=k-1$, we can count $k$-term arithmetic progressions in $A$ more or less as in the random case for $k=3$. Define

$$
\Lambda_{k}\left(f_{1}, \ldots, f_{k}\right)=N^{-2} \sum_{x, r} f_{1}(x) f_{2}(x+r) \ldots f_{k}(x+(k-1) r),
$$

the obvious generalization of the $\Lambda_{3}$. Now write $A(x)=\delta+f^{A}(x)$ and expand $\Lambda_{k}(A, \ldots, A)$ accordingly. This produces the leading term $\Lambda_{k}(\delta, \ldots, \delta)=\delta^{k}$, plus $2^{k}-1$ error terms involving at least one copy of $f^{A}$. Gowers shows that the error terms are all bounded by $\left\|f^{A}\right\|_{U^{k-1}}$; hence if the latter is small enough, we are done.

It remains to address the "structured" case when $\left\|f^{A}\right\|_{U^{k-1}}$ is large. This is a much harder task than for $k=3$, as any hopes for a linear structure or periodicity are quickly dispelled by explicit examples. Specifically, if $A(x)$ correlates closely enough with a function of the form $e^{2 \pi i \phi(x)}$, where $\phi$ is a polynomial of degree $k-2$, then $A$ need not have any linear bias whatsoever, but on the other hand $\left\|f^{A}\right\|_{U^{k-1}}$ is large. The underlying principle here is that $\left\|e^{2 \pi i \phi(x)}\right\|_{U^{k-1}}$ is an exponential sum over the $(k-1)$-st iterated differences of $\phi(x)$. The latter vanish if $\phi$ is a polynomial of degree $k-2$, hence in this case there are no cancellations in the exponential sum 
and $\left\|e^{2 \pi i \phi(x)}\right\|_{U^{k-1}}=1$. We invite the reader to try to verify this for $k=4$ and $\phi(x)=x^{2} / N$.

But now at least we know the enemy a little bit better, and we can ask whether all functions with large $U^{k-1}$ norm have "polynomial bias" as described above. Is there an inverse theorem for the Gowers $U^{d}$ norms with $d>2$ ? For $d=3$, such a result was indeed obtained by Green and Tao [87], but only much later, and even then the actual statement is rather more complicated. This is not just for technical reasons: the $\phi$ in the conclusion, instead of a quadratic polynomial, could mimic a quadratic form in several variables. (Gowers [73], [74] was already aware of this issue, as were Furstenberg and Weiss in [68].) No Gowers inverse theorem is currently known for $d \geq 4$. $^{1}$

Fortunately, Gowers's proof of Szemerédi's theorem does not in fact require a "global" inverse theorem as described above. It suffices to find much weaker partial polynomial patterns, or prove a "local" inverse theorem. Gowers accomplishes this by identifying a weak "iterated additive structure" in $A$, then performing a procedure that could be described, for lack of a better word, as discrete integration. It is in this part of the proof that advanced tools from additive number theory, such as the theorems of Freiman and Balog-Szemerédi, become crucial. Once a partial polynomial pattern is found, an exponential sums argument produces a density increment on a subprogression of $\mathbb{Z}_{N}$. At last, the inductive step is complete.

\section{Arithmetic Progressions in the PRIMes}

6.1. The Green-Tao theorem. We finally turn to the Green-Tao theorem on arithmetic progressions in the primes [85]:

Theorem 6.1. For any $k \geq 3$, the primes contain infinitely many $k$-term arithmetic progressions.

It had long been expected, at least on a heuristic level, that Theorem 6.1 should hold. Crude asymptotics for prime numbers are often suggested by the random model, where the set $P$ of primes is modelled by a random set $X$ such that each $x \in \mathbb{N}$ belongs to $X$ independently with probability $(\log x)^{-1}$. This model predicts, for example, that the number of primes in $\{1, \ldots, N\}$ should be about $N / \log N$, which is consistent with the prime number theorem. But it is also capable of making more ambitious predictions:

- The probability that $x, x+2$ are both in $X$ is $(\log x)^{-2}$. The random model would thus imply the twin prime conjecture: there should be $N(\log N)^{-2}$ primes $p \in\{1, \ldots, N\}$ such that $p+2$ is also prime.

- If $x, r \leq N$, and if $k$ is small compared to $N$, the probability that $x$, $x+r, \ldots, x+(k-1) r$ are all in $X$ is about $(\log N)^{-k}$ on average. Hence there should be about $N^{2}(\log N)^{-k} k$-term arithmetic progressions of primes in $\{1, \ldots, N\}$.

The reality is that the primes are far from being randomly distributed. For instance, the events " $x$ is prime" and " $x+1$ is prime" are not independent - they are mutually exclusive if $x \neq 2$. Unlike a truly random set, the primes are not uniformly distributed between residue classes: there is only one even prime, only

\footnotetext{
[98].

${ }^{1}$ On the other hand, infinitary theorems of this type are known in ergodic theory; see Host-Kra
} 
one divisible by 3 , etc. Any reasonable theorem or conjecture on the asymptotic distribution of primes must account for such "obstructions to uniformity".

It is reasonable, however, to expect that the primes will be distributed as randomly as possible within those constraints. Hardy and Littlewood [92] made a far-reaching conjecture which is, roughly, a quantitative version of this statement. We will not give a precise formulation of the conjecture here (see e.g. Soundarajan [165] for a very accessible introduction), but suffice it to say that a special case of it yields the asymptotic formulae $\left(\gamma_{k}+o(1)\right) N^{2}(\log N)^{-k}$ for the number of $k$-term arithmetic progressions in the primes less than $N$, where $\gamma_{k}$ are explicitly computable constants (for example, $\gamma_{3} \approx 1.32032$ ). The Hardy-Littlewood conjecture implies the twin primes conjecture as well, again with explicit asymptotics, and much more.

It nonetheless came as a surprise when Theorem 6.1 was actually proved. Prior to the Green-Tao work, the problem appeared to be well out of reach, with very few partial results available. The $k=3$ case was resolved by van der Corput back in 1939 [191], and Heath-Brown [94] proved that there are infinitely many 4-term arithmetic progressions consisting of three primes and a number which has at most 2 prime divisors. Both results were significant in their own right, but neither gave any indication as to how the general case might be approached. As it turned out, the Green-Tao proof did not require much new information about the primes themselves. According to Ben Green [83],

Our main advance, then, lies not in our understanding of the primes but rather in what we can say about arithmetic progressions.

The strategy of [85] was to embed the primes in a sufficiently random background set in which they have positive density, then prove a "relative Szemerédi theorem" which applies in this setting. The latter - also dubbed "transference principle" to reflect its reliance on the standard Szemerédi theorem - is the main contribution of [85], and we describe it first.

Instead of sets $A$ of positive relative density in $\mathbb{Z}_{N}$, we consider functions $f$ and $\nu$ on $\mathbb{Z}_{N}$ such that $0 \leq f \leq \nu$ and $\sum_{x} f(x) \geq \delta \sum_{x} \nu(x)$. Here $f$ is the target function (which will later be supported on the primes), and $\nu$ is the background function. A key point is that $f$ and $\nu$ need not be bounded uniformly in $N$. We assume $\nu$ to be pseudorandom in the sense that it satisfies certain explicit (somewhat cumbersome to reproduce in full) correlation conditions depending on $k$. Verifying these conditions for the characteristic function of the primes would amount more or less to proving the Hardy-Littlewood conjecture; hence it is critical that our $\nu$ be easier to work with.

We wish to prove a Szemerédi theorem in this setting; more precisely, we need to estimate from below the quantity

$$
\sum_{x, r} f(x) f(x+r) f(x+2 r) \ldots f(x+(k-1) r),
$$

which counts the number of $k$-term arithmetic progressions in a set $A$ if $f$ is the characteristic function of it. The proof of this follows the rough outline of Furstenberg's ergodic proof of Szemerédi's theorem, but draws also on ideas of other authors, including Gowers [74], [76] and Host-Kra [98]. An inductive procedure is used to decompose $f$ into random and quasiperiodic parts $f_{U}$ and $f_{\perp}$, where $f_{\perp}$ 
is non-negative and bounded, and $f_{U}$ is unbounded but has a very small $U^{k-1}$ Gowers norm. The contribution of the random part to (6.1) is negligible. On the other hand, the "usual" Szemerédi theorem gives a bound from below on the contribution of the quasiperiodic part, and the result follows. This may sound like a reasonably simple outline, but the actual argument is quite breathtaking in its execution.

We now have to find appropriate functions $f$ and $\nu$. Let $f(n)=\log n$ if $n$ is prime, and 0 otherwise. This is very close to the von Mangoldt function in number theory. We then define $\nu$ to be a modification of the von Mangoldt function, supported on the almost primes (roughly, numbers which do not have small divisors). From a harmonic-analytic point of view, we are applying a "high-frequency cut-off": the Fourier transform of $\nu$ is much better behaved than that of $f$, which explains in part why functions such as $\nu$ can be so useful in sieving techniques.

As it happened, back in 2003 Dan Goldston and Cem Yıldırım had announced a major result on small gaps between prime numbers:

There are infinitely many prime pairs $p, p^{\prime}$ such that $p^{\prime}-p=$ $o(\log p)$.

By the prime number theorem, the gaps between subsequent primes have size about $\log p$ on average. While large deviations from the average are expected to occur (the twin primes conjecture asserts that there are infinitely many gaps of size 2), it turned out to be very difficult to confirm it rigorously. An error was found in the Goldston-Yıldırım proof a few months later and the main claims were withdrawn, but preprints with proofs of partial results remained in circulation.

Green and Tao in [85] credit Andrew Granville for pointing them to the preprint [72]. The correlation estimates therein, with relatively minor changes, were sufficient to prove that $\nu$ was pseudorandom in the sense of [85]. It is well known that Goldston and Yıldırım, joined by János Pintz, eventually completed their proof in [71]. An excellent overview of that story is in Soundarajan [165].

We must note that Green and Tao did not even attempt in [85] to address the underlying "hard" question of whether the primes are in fact randomly distributed in the sense of Hardy-Littlewood. They took a different route instead, showing that even if the primes were not randomly distributed, they would still have to contain arithmetic progressions of any finite length, courtesy of a Szemerédi-type theorem. The advantage of this approach is that it applies equally well to positive density subsets of the primes. The disadvantage is that it only produces a lower bound $c_{k} N^{2}(\log N)^{-k}$ on the number of progressions up to $N$ rather than exact asymptotics with the correct constant.

In the more recent paper [89], however, Green and Tao confront directly the Hardy-Littlewood conjecture, formulating a broad strategy applicable in "nondegenerate" cases. (For the present purpose, the twin primes and Goldbach conjectures are both degenerate.) The strategy is conditional on certain conjectural statements, the Gowers Inverse Conjecture and Möbius and Nilsequences Conjecture, both of which have been proven by Green and Tao to the extent needed to resolve the case of non-degenerate systems of 2 equations [87], [90], but for now remain open in their full generality. In particular, the combined results of [89], [87], [90] imply the Hardy-Littlewood asymptotic formula for the number of 4-term arithmetic progressions in the primes. A more detailed exposition of this work, 
including the quadratic Fourier analysis for systems of 2 equations, is in Green [82].

The Green-Tao theorem has inspired a variety of other results in ergodic theory and number theory, such as Frantzikinakis-Host-Kra [61] or Tao-Ziegler [188]. The focus of this note will remain on connections to harmonic analysis, and thus we return to restriction theory for the last time.

6.2. What goes around, comes around. Restriction estimates for exponential sums over sets of integers, as opposed to the continuous Fourier transform, were first derived by Bourgain in [12], [15]. In [15] and its sequels, they were applied to proving well-posedness and Strichartz estimates for nonlinear evolution equations, such as Schrödinger and Korteweg-de Vries, on the torus $\mathbb{T}^{d}$. They were then revisited in 2003 by Green in [79], a paper that directly inspired the work in GreenTao [85]. The main result of [79] is the following common generalization of the theorems of Roth [149] and van der Corput [191].

Theorem 6.2. Let $A$ be a set of positive relative density in the primes, i.e.

$$
\varlimsup_{N \rightarrow \infty} \frac{\left|A \cap P_{N}\right|}{\left|P_{N}\right|}>0,
$$

where $P_{N}$ denotes the set of primes less than $N$. Then $A$ contains infinitely many 3-term arithmetic progressions.

Green's argument foreshadows the Green-Tao theorem of [85] in its use of a transference principle, or a relative Roth theorem, to find arithmetic progressions in subsets of a sparse but sufficiently random set. As in Section 6.1, we will consider unbounded functions $0 \leq f(n) \leq \lambda(n)$, except that now the majorant $\lambda$ is supported on the primes (rather than almost primes) and $f$ is supported on $A$. We wish to prove lower bounds on (6.1) for $k=3$. In this context, the randomness of $\lambda$ means simply that $\lambda$ has small Fourier coefficients, as explained earlier in connection with Roth's theorem. The von Mangoldt function $\Lambda$, a natural candidate for $\lambda$, does not quite meet that requirement, but this can be fixed by restricting $\Lambda$ to the primes in an appropriate arithmetic subprogression (the same $W$-trick is also used in [85]).

The proof of the transference principle in [79] does not proceed along the same lines as that in [85]. Instead, Green uses a Fourier-analytic approach, subsequently developed further in Green-Tao [86] (see also Tao-Vu [187]). His main tool is the estimate

$$
\|\widehat{g \lambda}\|_{\ell^{p}\left(\mathbb{Z}_{N}\right)} \leq C(p)\|g\|_{\ell^{2}\left(\mathbb{Z}_{N}, d \lambda\right)}, p>2 .
$$

The Fourier transform here is discrete rather than continuous, and $\lambda$ is a density rather than a measure, but otherwise (6.2) has exactly the same form as the restriction estimate (2.10) for the sphere in $\mathbb{R}^{d}$. More than that: Green's proof of (6.2), as well as Bourgain's proof of a similar estimate for the "discrete sphere" in [15], follows very closely the Tomas-Stein argument in Euclidean harmonic analysis, from interpolation between the endpoints down to such details as the use of a Littlewood-Paley dyadic decomposition. The Fourier-analytic estimates on $\lambda$ needed in the proof of (6.2), along with similar estimates for the almost primes, are derived via the circle method, a classic technique in analytic number theory which had also been used by van der Corput.

Do the primes have the shape of a sphere? Most certainly not. Harmonic analysts are often quick to associate the restriction phenomenon with curvature, but 
restriction estimates are also known in other Euclidean settings, such as sets of fractional dimension (Mockenhaupt [129], Wolff [196]). Time will tell whether the analogy can be usefully pursued further.

There are certainly many more links between analysis, number theory and combinatorics waiting to be discovered. We are only beginning, for instance, to explore the possibility of applying methods of additive combinatorics to problems in analysis. It seems safe to say that the areas of research described here will remain active for a long time to come.

\section{FURTHER READING}

Sections 1-3. Background information on the Kakeya problem and the details of a Kakeya set construction can be found in Besicovitch [6], Davis-Chang [42], Falconer [58], Stein [169], and Wolff [197], [200].

Stein's book [169] is a comprehensive, if somewhat overwhelming, reference on the harmonic analytic questions in Section 2, including the restriction problem, averaging and maximal estimates, and many other closely related problems which we were not able to discuss here. The monographs by Davis-Chang [42] and Wolff [200] cover less material but are more accessible to a reader less familiar with the subject. These references were all published before the latest rush of work in the area; hence we refer the reader to Katz-Tao [115] and Tao [177] for updated information on the Kakeya and restriction problems, respectively. Connections to PDE problems are highlighted in Sogge [157], Stein [169], and Tao [181].

The historical information in Section 1 comes from a variety of sources, including Besicovitch's article [6], Kenneth Falconer's historical comments in [58], and the Internet-based MacTutor History of Mathematics Archive, maintained at the University of St. Andrews (http://www-history.mcs.st-andrews.ac.uk/history), from where I borrowed the quote by A.A. Friedmann in Subsection 1.1.

Section 4. Pach-Agarwal [139] and Matousek [125] are standard and comprehensive references on combinatorial geometry, and the survey article by Pach and Sharir [141] focuses specifically on incidence problems. Applications of combinatorial geometry in harmonic analysis are discussed e.g. in Schlag [154] and Wolff [197].

Sections 5-6. A comprehensive up-to-date survey of additive combinatorics is in Tao-Vu [187]; general expository articles include Croot-Lev [39], Gowers [75], Granville [78], and Ruzsa [153]. Additive number theory, including Fourier-analytic methods, Freiman's theorem and inverse problems, is surveyed in Nathanson [135]. For more on sum-product problems, see Bourgain [22], Chang [33], and Elekes [47]. There are many surveys and expositions of Szemerédi's theorem and the Green-Tao theorem, including Green [82], [83], Kra [119], and Tao [178], [180], [182], [187].

\section{ABout THE AUThor}

Izabella Łaba is a professor of mathematics at the University of British Columbia. She has received the Coxeter-James and Krieger-Nelson Prizes of the Canadian Mathematical Society. 


\section{ACKNOWLEDGEMENTS}

My knowledge and understanding of the research areas described here were shaped in part by the conversations I have had with many people over the years. In particular, I would like to thank Andrew Granville, Ben Green, Alex Iosevich, Nets Katz, Michael Lacey, Wilhelm Schlag, Carol Shubin, Christopher Sogge, Jozsef Solymosi, Terence Tao, and Thomas Wolff. I am also grateful to Mariah Hamel, Michael Lacey, and the anonymous referees for helpful comments and suggestions.

\section{REFERENCES}

1. B. Aronov, M. Sharir, Cutting circles into pseudo-segments and improved bounds on the number of incidences, Discrete Comput. Geom. 28 (2002), 475-490. MR1949895 (2004i:52011)

2. A. Balog, E. Szemerédi, A statistical theorem of set addition, Combinatorica 14 (1994), 263-268. MR1305895 (95m:11019)

3. M.D. Bateman, N.H. Katz, Kakeya sets in Cantor directions, preprint, 2006.

4. A.S. Besicovitch, Sur deux questions d'intégrabilité des fonctions, J. Soc. Phys.-Math. (Perm), 2 (1919), 105-123.

5. A.S. Besicovitch, On Kakeya's problem and a similar one, Math. Zeitschrift 27 (1928), 312-320. MR1544912

6. A.S. Besicovitch, The Kakeya problem, Amer. Math. Monthly 70 (1963), 697-706. MR0157266 (28:502)

7. A.S. Besicovitch, On fundamental geometric properties of plane line-sets, J. London Math. Soc. 39 (1964), 441-448. MR0171896 (30:2122)

8. V. Bergelson, B. Host, B. Kra, Multiple recurrence and nilsequences (with an appendix by I. Ruzsa), Invent. Math. 160 (2005), 261-303. MR2138068 (2007i:37009)

9. V. Bergelson, A. Leibman, Polynomial extensions of van der Waerden's and Szemerédi's theorems, J. Amer. Math. Soc. 9 (1996), 725-753. MR1325795 (96j:11013)

10. Y. Bilu, Structure of sets with small sumset, in Structure Theory of Set Addition, Astérisque 258 (1999), 77-108. MR1701189 (2000h:11109)

11. J. Bourgain, Averages in the plane over convex curves and maximal operators, J. Analyse Math. 47 (1986), 69-85. MR874045 (88f:42036)

12. J. Bourgain, On $\Lambda(p)$-subsets of squares, Israel J. Math. 67 (1989), 291-311. MR1029904 (91d:43018)

13. J. Bourgain, Besicovitch type maximal operators, and applications to Fourier analysis, Geom. Funct. Anal. 1 (1991), 147-187. MR1097257 (92g:42010)

14. J. Bourgain, $L^{p}$ estimates for oscillatory integrals in several variables, Geom. Funct. Anal. 1 (1991), 321-374. MR1132294 (93e:42021)

15. J. Bourgain, Fourier restriction phenomena for certain lattice subsets and applications to nonlinear evolution equations, I, Geom. Funct. Anal. 3 (1993), 107-156. MR1209299 (95d:35160a)

16. J. Bourgain, Hausdorff dimension and distance sets, Israel J. Math. 87 (1994), 193-201. MR1286826 (95h:28008)

17. J. Bourgain, On the dimension of Kakeya sets and related maximal inequalities, Geom. Funct. Anal. 9 (1999), 256-282. MR1692486 (2000b:42013)

18. J. Bourgain, On triples in arithmetic progression, Geom. Funct. Anal. 9 (1999), 968-984. MR1726234 (2001h:11132)

19. J. Bourgain, On the Erdös-Volkmann and Katz-Tao ring conjectures, Geom. Funct. Anal. 13 (2003), 334-365. MR1982147 (2004d:11070)

20. J. Bourgain, Mordell's exponential sum revisited, J. Amer. Math. Soc. 18 (2005), 477-499. MR2137982 (2006b:11099)

21. J. Bourgain, More on the sum-product phenomenon in prime fields and its applications, Int. J. Number Theory 1 (2005), 1-32. MR2172328 (2006g:11041)

22. J. Bourgain, New encounters in combinatorial number theory: from the Kakeya problem to cryptography, Perspectives in analysis, 17-26, Math. Phys. Stud. 27, Springer, Berlin, 2005. MR2206765 (2006m:11030)

23. J. Bourgain, Roth's theorem on progressions revisited, preprint, 2007. 
24. J. Bourgain, M.-C. Chang, On the size of $k$-fold sum and product sets of integers, J. Amer. Math. Soc. 17 (2004), 473-497. MR2051619 (2005c:11024)

25. J. Bourgain, A. Gamburd, P. Sarnak, Sieving and expanders, C. R. Math. Acad. Sci. Paris, Ser. I, 343 (2006), 155-159. MR2246331 (2007b:11139)

26. J. Bourgain, A.A. Glibichuk, S.V. Konyagin, Estimates for the number of sums and products and for exponential sums in fields of prime order, J. London Math. Soc. 73 (2006), 380-398. MR2225493 (2007e:11092)

27. J. Bourgain, N.H. Katz, T. Tao, A sum-product estimate in finite fields, and applications, Geom. Funct. Anal. 14 (2004), 27-57. MR2053599 (2005d:11028)

28. H. Busemann, W. Feller, Differentiation der L-integrale, Fund. Math. 22 (1934), 226-256.

29. A. Carbery, M. Christ, J. Wright, Multidimensional van der Corput and sublevel set estimates, J. Amer. Math. Soc. 12 (1999), 981-1015. MR1683156 (2000h:42010)

30. M.-C. Chang, A polynomial bound in Freiman's theorem, Duke Math. J. 3 (2002), 399-419. MR1909605 (2003d:11151)

31. M.-C. Chang, Factorization in generalized arithmetic progressions and applications to the Erdös-Szemerédi sum-product problems, Geom. Funct. Anal. 13 (2003), 720-736. MR2006555 (2004g:11007)

32. M.-C. Chang, Erdős-Szemerédi problem on sum set and product set, Ann. Math. 157 (2003), 939-957. MR1983786 (2004c:11026)

33. M.-C. Chang, Some problems in combinatorial number theory, preprint, 2007, to appear in Integers: Electronic Journal of Combinatorial Number Theory.

34. M. Christ, Estimates for the k-plane transform, Indiana Univ. Math. J. 33 (1984), 891-910. MR763948 (86k:44004)

35. M. Christ, Convolution, curvature, and combinatorics. A case study, Internat. Math. Research Notices 19 (1998), 1033-1048. MR1654767 (2000a:42026)

36. M. Christ, A. Nagel, E.M. Stein, S. Wainger, Singular and maximal Radon transforms: Analysis and geometry, Ann. Math. 150 (1999), 489-577. MR1726701 (2000j:42023)

37. K. Clarkson, H. Edelsbrunner, L. Guibas, M. Sharir, E. Welzl, Combinatorial complexity bounds for arrangements of curves and spheres, Discrete Comput. Geom. 5 (1990), 99-160. MR1032370 (91f:52021)

38. A. Córdoba, The Kakeya maximal function and spherical summation multipliers, Amer. J. Math. 99 (1977), 1-22. MR0447949 (56:6259)

39. E. Croot, V. Lev, Open problems in additive combinatorics, to appear in Proceedings of a School in Additive Combinatorics, Montreal, March 30th-April 5th, 2006, eds: A. Granville, M. Nathanson and J. Solymosi.

40. F. Cunningham, The Kakeya problem for simply connected and for star-shaped sets, Amer. Math. Monthly 78 (1971), 114-129. MR0275287 (43:1044)

41. R.O. Davies, Some remarks on the Kakeya problem, Proc. Cambridge Phil. Soc. 69 (1971), 417-421. MR0272988 (42:7869)

42. K.M. Davis, Y.C. Chang, Lectures on Bochner-Riesz Means, London Math. Soc. Lecture Notes 114, Cambridge Univ. Press, Cambridge, 1987. MR921849 (88m:42031)

43. S. Drury, $L^{p}$ estimates for the $x$-ray transform, Ill. J. Math. 27 (1983), 125-129. MR684547 (85b:44004)

44. G.A. Edgar, C. Miller, Borel subrings of the reals, Proc. Amer. Math. Soc. 131 (2003), 1121-1129. MR1948103 (2004d:28017)

45. G. Elek, B. Szegedy, Limits of Hypergraphs, Removal and Regularity Lemmas. A Nonstandard Approach, preprint.

46. G. Elekes, On the number of sums and products, Acta Arith. 81 (1997), 365-367. MR1472816 (98h:11026)

47. G. Elekes, Sums versus product in algebra, number theory and Erdös geometry, unpublished preprint, 2001.

48. G. Elekes, I.Z. Ruzsa, Few sums, many products, Studia Sci. Math. Hungar. 40 (2003), 301-308. MR2036961 (2005d:11011)

49. G. Elekes, Cs. Tóth, Incidences of not too degenerate hyperplanes, Proc. 21st ACM Sympos. Comput. Geom. (Pisa, 2005), ACM Press, 16-21.

50. B. Erdog̃an, A bilinear Fourier extension theorem and applications to the distance set problem, Internat. Math. Res. Notices 23 (2005), 1411-1425. MR2152236 (2006h:42020) 
51. B. Erdog̃an, On Falconer's distance set conjecture, Rev. Mat. Iberoamericana 22 (2006), 649-662. MR2294792

52. P. Erdős, On sets of distances of $n$ points, Amer. Math. Monthly 53 (1946), 248-250. MR0015796 (7:471c)

53. P. Erdős, E. Szemerédi, On sums and products of integers, Studies in Pure Mathematics: To the Memory of Paul Turán, Birkhäuser, Basel, 1983, pp. 213-218. MR820223 (86m:11011)

54. P. Erdős, P. Turán, On some sequences of integers, J. London Math. Soc. 16 (1936), 261-264.

55. P. Erdős, B. Volkmann, Additive Gruppen mit vorgegebener Hausdorffscher Dimension, J. Reine Angew. Math. 221 (1966), 203-208. MR0186782 (32:4238)

56. K.J. Falconer, Rings of fractional dimension, Mathematika 31 (1984), 25-27. MR762173 (85m:28004)

57. K. J. Falconer, On the Hausdorff dimensions of distance sets, Mathematika 32 (1985), 206212. MR834490 (87j:28008)

58. K.J. Falconer, The Geometry of Fractal Sets, Cambridge Univ. Press, Cambridge, 1986. MR867284 (88d:28001)

59. C. Fefferman, Inequalities for strongly singular operators, Acta Math. 124 (1970), 9-36. MR0257819 (41:2468)

60. C. Fefferman, The multiplier problem for the ball, Ann. Math. 94 (1971), 330-336. MR0296602 (45:5661)

61. N. Frantzikinakis, B. Host, B. Kra, Multiple recurrence and convergence for sequences related to the prime numbers, J. Reine Angew. Math., to appear.

62. N. Frantzikinakis, B. Kra, Convergence of multiple ergodic averages for some commuting transformations, Ergodic Theory Dynamical Systems 25 (2005), 799-809. MR2142946 (2007b:37009)

63. G.A. Freiman, On the addition of finite sets (in Russian), Dokl, Akad. Nauk SSSR 158 (1964), 1038-1041. MR0168529 (29:5791)

64. G.A. Freiman, Foundations of a Structural Theory of Set Addition (translated from Russian), Translations of Mathematical Monographs, vol. 37, Amer. Math. Soc., 1973. MR0360496 (50:12944)

65. M. Fujiwara, S. Kakeya, On some problems of maxima and minima for the curve of constant breadth and the in-revolvable curve of the equilateral triangle, Tôhoku Mathematical Journal 11 (1917), 92-110.

66. H. Furstenberg, Ergodic behaviour of diagonal measures and a theorem of Szemerédi on arithmetic progressions, J. Analyse Math. 31 (1977), 204-256. MR0498471 (58:16583)

67. H. Furstenberg, Y, Katznelson, An ergodic Szemerédi theorem for commuting transformations, J. Analyse Math. 34 (1978), 275-291. MR531279 (82c:28032)

68. H. Furstenberg, B. Weiss, A mean ergodic theorem for $1 / N \sum_{n=1}^{N} f\left(T^{n} x\right) g\left(T^{n^{2}} x\right)$, in Convergence in Ergodic Theory and Probability, Ohio State Univ. Math. Res. Inst. Publ. 5, Walter de Gruyter \& Co, Berlin, 1996, 193-227. MR1412607 (98e:28019)

69. M.Z. Garaev, An explicit sum-product estimate in $\mathbb{F}_{p}$, preprint, 2007.

70. G. Garrigós, A. Seeger, On plate decompositions of cone multipliers, Proceedings of the conference on Harmonic Analysis and Its Applications, Hokkaido University, Sapporo, 2005.

71. D. Goldston, J. Pintz, C.Y. Ylldırım, Primes in tuples I, Ann. Math., to appear.

72. D. Goldston, C.Y. Yıldırım, Higher correlations of divisor sums related to primes III: Small gaps between primes, preprint, 2004.

73. W.T. Gowers, A new proof of Szemerédi's theorem for arithmetic progressions of length four, Geom. Funct. Anal. 8 (1998), 529-551. MR1631259 (2000d:11019)

74. W.T. Gowers, A new proof of Szemerédi's theorem, Geom. Funct. Anal. 11 (2001), 465-588. MR1844079 (2002k:11014)

75. W.T. Gowers, Some unsolved problems in additive and combinatorial number theory, preprint, 2001 (available at http://www.dpmms.cam.ac.uk/ ${ }^{\sim}$ wtg10/papers.html).

76. W. T. Gowers, Hypergraph regularity and the multidimensional Szemerédi theorem, preprint, 2005.

77. W. T. Gowers, Quasirandomness, counting and regularity for 3-uniform hypergraphs, Combin. Probab. Comput. 15 (2006), 143-184. MR2195580

78. A. Granville, An introduction to additive combinatorics, to appear in Proceedings of a School in Additive Combinatorics, Montreal, March 30th-April 5th, 2006, eds: A. Granville, M. Nathanson and J. Solymosi. 
79. B. Green, Roth's Theorem in the primes, Ann. Math. 161 (2005), 1609-1636. MR2180408 (2007a:11136)

80. B. Green, Structure theory of set addition, unpublished, available at http://www.dpmms.cam.ac.uk/〜bjg23/papers/icmsnotes.pdf.

81. B. Green, Finite field models in additive combinatorics, in Surveys in Combinatorics, London Math. Soc. Lecture Notes 327 (2005), 1-29. MR2187732 (2006j:11030)

82. B. Green, Generalising the Hardy-Littlewood method for primes, Proceedings of the International Congress of Mathematicians, Vol. II, 373-399, Eur. Math. Soc., Zürich, 2006. MR2275602

83. B. Green, Long arithmetic progressions of primes, submitted to Proceedings of the GaussDirichlet conference, Göttingen, 2005.

84. B. Green, I.Z. Ruzsa, Freiman's theorem in an arbitrary abelian group, J. London Math. Soc. 75 (2007), 163-175. MR2302736

85. B. Green, T. Tao, The primes contain arbitrarily long arithmetic progressions, Ann. Math., to appear.

86. B. Green, T. Tao, Restriction theory of the Selberg sieve, with applications, J. Théor. Nombres Bordeaux 18 (2006), 147-182. MR2245880

87. B. Green, T. Tao, An inverse theorem for the Gowers $U^{3}(G)$ norm, Proc. Edin. Math. Soc., to appear.

88. B. Green, T. Tao, Compressions, convex geometry, and the Freiman-Bilu theorem, Q. J. Math. 57 (2006), 495-504. MR2277597 (2007g:11013)

89. B. Green, T. Tao, Linear equations in primes, Ann. Math., to appear.

90. B. Green, T. Tao, Quadratic uniformity of the Möbius function, preprint, 2006.

91. B. Green, T. Tao, New bounds for Szemerédi's theorem, II. A new bound for $r_{4}(N)$, preprint, 2006.

92. G. Hardy, J. Littlewood, Some problems of "partitio numerorum", III: On the expression of a number as a sum of primes, Acta Math. 44 (1923), 1-70. MR1555183

93. G. Hardy, J. Littlewood, A maximal theorem with function-theoretic applications, Acta Math. 54 (1930), 81-116. MR1555303

94. D.R. Heath-Brown, Three primes and an almost prime in arithmetic progression, J. London Math. Soc. 23 (1981), 396-414. MR616545 (82j:10074)

95. D.R. Heath-Brown, Integer sets containing no arithmetic progressions, J. London Math. Soc. 35 (1987), 385-394. MR889362 (88g:11005)

96. C.S. Herz, Fourier transforms related to convex sets, Ann. Math. 75 (1962), 81-92. MR0142978 (26:545)

97. L. Hörmander, The Analysis of Linear Partial Differential Operators, volume 1, 2nd edition, Springer Verlag, 1990. MR1065136 (91m:35001b)

98. B. Host, B. Kra, Nonconventional ergodic averages and nilmanifolds, Ann. Math. 161 (2005), 397-488. MR2150389 (2007b:37004)

99. A. Iosevich, Curvature, combinatorics and the Fourier transform, Notices Amer. Math. Soc. 48 (2001), 577-583. MR1834352 (2002e:42004)

100. A. Iosevich, D. Hart. J. Solymosi, Sum-product estimates in finite fields, Internat. Math. Res. Notices, to appear.

101. A. Iosevich, S. Hofmann, Circular averages and Falconer/Erdös distance conjecture in the plane for random metrics, Proc. Amer. Mat. Soc. 133 (2005), 133-143. MR2085162 (2005k:42031)

102. A. Iosevich, H. Jorati, I. Łaba, Geometric incidence theorems via Fourier analysis, preprint, 2007.

103. A. Iosevich, N.H. Katz, T. Tao, The Fuglede spectral conjecture holds for convex bodies in the plane, Math. Res. Letters 10 (2003), 559-570. MR2024715 (2004i:42020)

104. A. Iosevich, I. Laba, K-distance sets, Falconer conjecture, and discrete analogs, Integers: Electronic Journal of Combinatorial Number Theory 5 (2005), \#A08 (hardcopy in: Topics in Combinatorial Number Theory: Proceedings of the Integers Conference 2003 in Honor of Tom Brown, DIMATIA, ITI Series, vol. 261). MR2192086 (2006i:42033)

105. J.P. Kahane, Trois notes sur les ensembles parfaites lineaires, L'enseignement Mathematique Revue Internationale 15 (1969), 185-192. MR0245734 (39:7040)

106. S. Kakeya, Some problems on minima and maxima regarding ovals, Tôhoku Science Reports, 6 (1917), 71-88. 
107. N.H. Katz, A counterexample for maximal operators over a Cantor set of directions, Math. Res. Lett. 3 (1996), 527-536. MR1406017 (98b:42032)

108. N.H. Katz, Elementary proofs and the sums differences problem, Collect. Math. 2006, Vol. Extra, 275-280. MR2264213 (2007g:42019)

109. N.H. Katz, I. Łaba, T. Tao, An improved bound on the Minkowski dimension of Besicovitch sets in $\mathbb{R}^{3}$, Ann. of Math. 152 (2000), 383-446. MR1804528 (2002i:28006)

110. N.H. Katz, C.-Y. Shen, A slight improvement to Garaev's sum-product estimate, preprint, 2007.

111. N.H. Katz, C.-Y. Shen, Garaev's inequality in fields not of prime order, preprint, 2007.

112. N.H. Katz, T. Tao, Bounds on arithmetic projections, and applications to the Kakeya conjecture, Math. Res. Letters 6 (1999), 625-630. MR1739220 (2000m:28006)

113. N.H. Katz, T. Tao, Some connections between Falconer's distance set conjecture and sets of Furstenberg's type, New York J. Math. 7 (2001), 149-187. MR1856956 (2002i:28013)

114. N.H. Katz, T. Tao, New bounds for Kakeya sets, J. Anal. Math. 87 (2002), 231-263. MR1945284 (2003i:28006)

115. N.H. Katz, T. Tao, Recent progress on the Kakeya conjecture, Publicacions Matematiques, Proceedings of the 6th El Escorial International Conference on Harmonic Analysis and Partial Differential Equations, U. Barcelona, 2002, 161-180. MR1964819 (2003m:42036)

116. N.H. Katz, G. Tardos, A new entropy inequality for the Erdös distance problem, in Towards a Theory of Geometric Graphs (J. Pach, ed.), Contemporary Mathematics, vol. 342, Amer. Math. Soc., 2004. MR2065258 (2005f:52033)

117. L. Kolasa, T. Wolff, On some variants of the Kakeya problem, Pacific J. Math. 190 (1999), 111-154. MR1722768 (2001g:42032)

118. M.N. Kolountzakis, Distance sets corresponding to convex bodies, Geom. Funct. Anal. 14 (2004), 734-744. MR2084977 (2005g:52009)

119. B. Kra, The Green-Tao theorem on arithmetic progressions in the primes: an ergodic point of view, Bull. Amer. Math. Soc. 43 (2006), 3-23. MR2188173 (2006h:11113)

120. I. Łaba, J. Solymosi, Incidence theorems for pseudoflats, Discrete Comput. Geom. 37 (2007), 163-174. MR2295051

121. I. Łaba, T. Tao, An improved bound for the Minkowski dimension of Besicovitch sets in medium dimension, Geom. Funct. Anal. 11 (2001), 773-806. MR1866801 (2003b:28006)

122. I. Łaba, T. Wolff, A local smoothing estimate in higher dimensions, J. d'Analyse Math. 88 (2002), 149-171. MR1956533 (2005b:35015)

123. A. Leibman, Convergence of multiple ergodic averages along polynomials of several variables, Israel J. Math. 146 (2005), 303-316. MR2151605 (2006c:28016)

124. J.M. Marstrand, Packing circles in the plane, Proc. London Math. Soc. 55 (1987), 37-58. MR887283 (88i:28012)

125. J. Matousek, Lectures on discrete geometry, Springer-Verlag, 2002. MR1899299 (2003f:52011)

126. P. Mattila, Spherical averages of Fourier transforms of measures with finite energy: dimensions of intersections and distance sets, Mathematika 34 (1987), 207-228. MR933500 (90a:42009)

127. P. Mattila, Geometry of sets and measures in Euclidean spaces, Cambridge Studies in Advanced Mathematics, vol. 44, Cambridge University Press, 1995. MR1333890 (96h:28006)

128. W.P. Minicozzi, C.D. Sogge, Negative results for Nikodym maximal functions and related oscillatory integrals in curved space, Math. Res. Lett. 4 (1997), 221-237. MR1453056 (98k:42025)

129. G. Mockenhaupt, Salem sets and restriction properties of Fourier transforms, Geom. Funct. Anal. 10 (2000), 1579-1587. MR1810754 (2001m:42026)

130. G. Mockenhaupt, A. Seeger, C.D. Sogge, Wave front sets, local smoothing and Bourgain's circular maximal theorem, Ann. of Math. 136 (1992), 207-218. MR1173929 (93i:42009)

131. G. Mockenhaupt, T. Tao, Kakeya and restriction phenomena for finite fields, Duke Math. J. 121 (2004), 35-74. MR2031165 (2004m:11200)

132. A. Moyua, A. Vargas, L. Vega, Restriction theorems and maximal operators related to oscillatory integrals in $\mathbb{R}^{3}$, Duke Math. J. 96 (1999), 547-574. MR1671214 (2000b:42017)

133. A. Nagel, E.M. Stein, S. Wainger, Differentiation in lacunary directions, Proc. Nat. Acad. Sci. U.S.A. 75 (1978), 1060-1062. MR0466470 (57:6349) 
134. B. Nagle, V. Rödl, M. Schacht, The counting lemma for regular k-uniform hypergraphs, Random Structures Algorithms 28 (2006), 113-179. MR2198495 (2007d:05084)

135. M.B. Nathanson, Additive Number Theory: Inverse Problems and the Geometry of Sumsets, Graduate Texts in Mathematics 165, Springer-Verlag, 1996. MR1477155 (98f:11011)

136. O. Nikodym, Sur les ensembles accessibles, Fund. Math. 10 (1927), 116-168.

137. D. Oberlin, E.M. Stein, Mapping properties of the Radon transform, Indiana U. Math. J. 31 (1982), 641-650. MR667786 (84a:44002)

138. R. Oberlin, Two bounds on the $x$-ray transform, preprint, 2006.

139. J. Pach, P.K. Agarval, Combinatorial geometry, John Wiley \& Sons, New York, NY, 1995. MR1354145 (96j:52001)

140. J. Pach, M. Sharir, On the number of incidences between points and curves, Combinatorics, Probability and Computing 7 (1998), 121-127. MR1611057 (99b:52037)

141. J. Pach, M. Sharir, Geometric incidences, in Towards a Theory of Geometric Graphs (J. Pach, ed.), Contemporary Mathematics, vol. 342, Amer. Math. Soc., 2004. MR2065247 (2004m:05004)

142. J. Pál, Ein Minimumproblem für Ovale, Math. Ann. 83 (1921), 311-319. MR1512015

143. D.H. Phong, E.M. Stein, Hilbert integrals, singular integrals, and Radon transforms I. Acta Math. 157 (1986), 99-157. MR857680 (88i:42028a)

144. D.H. Phong, E.M. Stein, Hilbert integrals, singular integrals, and Radon transforms. II. Invent. Math. 86 (1986), 75-113. MR0853446 (88i:42028b)

145. M. Pramanik, A. Seeger, $L^{p}$ regularity of averages over curves and bounds for associated maximal operators, Amer. J. Math. 129 (2007), 61-103. MR2288738

146. V. Rödl, M. Schacht, Regular partitions of hypergraphs, to appear.

147. V. Rödl, J. Skokan, Regularity lemma for $k$-uniform hypergraphs, Random Structures and Algorithms 25 (2004), 1-42. MR2069663 (2005d:05144)

148. V. Rödl, J. Skokan, Applications of the regularity lemma for uniform hypergraphs, Random Structures and Algorithms 28 (2006), 180-194. MR2198496 (2006j:05099)

149. K. Roth, On certain sets of integers, J. London Math. Soc. 28 (1953), 104-109. MR0051853 $(14: 536 \mathrm{~g})$

150. I.Z. Ruzsa, An application of graph theory to additive number theory, Scientia, Ser. A 3 (1989), 97-109. MR2314377

151. I.Z. Ruzsa, Arithmetic progressions and the number of sums, Period. Math. Hung. 25 (1992), 105-111. MR1200845 (94a:11019)

152. I.Z. Ruzsa, Generalized arithmetic progressions and sumsets, Acta Math. Hungar. 65 (1994), 379-388. MR1281447 (95k:11011)

153. I.Z. Ruzsa, Additive combinatorics and geometry of numbers, Proceedings of the International Congress of Mathematicians, Vol. III, 911-930, Eur. Math. Soc., Zürich, 2006. MR2275712

154. W. Schlag, On continuum incidence problems related to harmonic analysis, J. Funct. Anal. 201 (2003), 480-521. MR1986697 (2004k:43009)

155. W. Schlag, A geometric proof of the circular maximal theorem, Duke. J. Math. 93 (1998), 505-533. MR1626711 (99d:42039)

156. I.D. Shkredov, On a generalization of Szemerédi's theorem, Proc. London Math. Soc. 93 (2006), 723-760. MR2266965 (2007i:11018)

157. C.D. Sogge, Fourier Integrals in Classical Analysis, Cambridge University Press, Cambridge, 1993. MR1205579 (94c:35178)

158. C.D. Sogge, Smoothing estimates for the wave equation and applications, in Proceedings of the ICM, Zurich 1994, vol. 1, 896-906, Birkhäuser, Basel, 1995. MR1403989 (97d:35123)

159. J. Solymosi, A note on a question of Erdös and Graham, Combin. Probab. Comput. 13 (2004), 263-267. MR2047239 (2004m:11012)

160. J. Solymosi, On the number of sums and products, Bull. London Math. Soc. 37 (2005), 491-494. MR2143727 (2006c:11021)

161. J. Solymosi, Sums and products of complex numbers, J. Théor. Nombres Bordeaux 17 (2005), 921-924. MR2212132 (2006m:11011)

162. J. Solymosi, Cs. Tóth, Distinct distances in the plane, Discrete Comput. Geom. 25 (2001), 629-634. MR1838423 (2002c:52020)

163. J. Solymosi, Cs. Tóth, Distinct distances in homogeneous sets in Euclidean space, Discrete Comput. Geom. 35 (2006), 537-549. MR2225673 (2007c:52020) 
164. J. Solymosi, V. Vu, Distinct distances in high dimensional homogeneous sets in Towards a Theory of Geometric Graphs (J. Pach, ed.), Contemporary Mathematics, vol. 342, Amer. Math. Soc., 2004 MR2065269 (2005m:52026)

165. K. Soundarajan, Small gaps between prime numbers: The work of Goldston-Pintz-Yıldırım, Bull. Amer. Math. Soc. 44 (2007), 1-18. MR2265008

166. J. Spencer, E. Szemerédi, W. Trotter, Unit distances in the Euclidean plane, in Graph Theory and Combinatorics, B. Bollobás, ed., Academic Press, London, 1984, 293-303. MR777185 (86m:52015)

167. E.M. Stein, Maximal functions: Spherical means, Proc. Nat. Acad. Sci. U.S.A. 73 (1976), 2174-2175. MR0420116 (54:8133a)

168. E.M. Stein, Oscillatory integrals in Fourier analysis, in Beijing Lectures in Harmonic Analysis (E.M. Stein, ed.), Ann. Math. Stud. \#112, Princeton Univ. Press, 1986, pp. 307-355. MR864375 (88g:42022)

169. E.M. Stein, Harmonic Analysis, Princeton Univ. Press, Princeton, 1993. MR1232192 (95c:42002)

170. R. Strichartz, Restrictions of Fourier transforms to quadratic surfaces and decay of solutions of wave equations, Duke Math. J. 44 (1977), 705-714. MR0512086 (58:23577)

171. L. Székely, Crossing numbers and hard Erdős problems in discrete geometry, Combinatorics, Probability, and Computing 6 (1997), 353-358. MR1464571 (98h:52030)

172. E. Szemerédi, On sets of integers containing no four elements in arithmetic progression, Acta Math. Acad. Sci. Hungar. 20 (1969), 89-104. MR0245555 (39:6861)

173. E. Szemerédi, On sets of integers containing no $k$ elements in arithmetic progression, Acta Arith. 27 (1975), 199-245. MR0369312 (51:5547)

174. E. Szemerédi, Integer sets containing no arithmetic progressions, Acta Math. Hungar. 56 (1990), 155-158. MR1100788 (92c:11100)

175. E. Szemerédi, W.T. Trotter, Extremal problems in discrete geometry, Combinatorica 3 (1983), 381-392. MR729791 (85j:52014)

176. T. Tao, A sharp bilinear restriction estimate for paraboloids, Geom. Funct. Anal. 13 (2003), 1359-1384. MR2033842 (2004m:47111)

177. T. Tao, Recent progress on the restriction conjecture, to appear in Park City conference proceedings.

178. T. Tao, Arithmetic progressions and the primes, Collect. Math. (2006), Vol. Extra, 37-88. MR2264205

179. T. Tao, A variant of the hypergraph removal lemma, J. Combin. Theory Ser. A 113 (2006), 1257-1280. MR2259060

180. T. Tao, The dichotomy between structure and randomness, arithmetic progressions, and the primes, Proceedings of the International Congress of Mathematicians, Vol. I, Eur. Math. Soc., Zürich, 2006.

181. T. Tao, Nonlinear dispersive equations: local and global analysis, CBMS Regional Conference Series in Mathematics, Amer. Math. Soc., 2006. MR2233925

182. T. Tao, What is good mathematics?, Bull. Amer. Math. Soc. 44 (2007), 623-634.

183. T. Tao, Norm convergence of multiple ergodic averages for commuting transformations, preprint, 2007.

184. T. Tao, A. Vargas, L. Vega, A bilinear approach to the restriction and Kakeya conjectures, J. Amer. Math. Soc. 11 (1998), 967-1000. MR1625056 (99f:42026)

185. T. Tao, A. Vargas, A bilinear approach to cone multipliers I. Restriction estimates, Geom. Funct. Anal. 10 (2000), 185-215. MR1748920 (2002e:42012)

186. T. Tao, A. Vargas, A bilinear approach to cone multipliers II. Applications, Geom. Funct. Anal. 10 (2000), 216-258. MR1748921 (2002e:42013)

187. T. Tao, V. Vu, Additive Combinatorics, Cambridge University Press, 2006. MR2289012

188. T. Tao, T. Ziegler, The primes contain arbitrarily long polynomial progressions, Acta Math., to appear.

189. P.A. Tomas, A restriction theorem for the Fourier transform, Bull. Amer. Math. Soc. 81 (1975), 477-478. MR0358216 (50:10681)

190. P.A. Tomas, Restriction theorems for the Fourier transform, in Harmonic Analysis in Euclidean Spaces, G. Weiss and S. Wainger, eds., Proc. Symp. Pure Math. \#35, Amer. Math. Soc., 1979, vol. I, pp. 111-114. MR545245 (81d:42029) 
191. J.G. van der Corput, Über Summen von Primzahlen und Primzahlquadraten, Math. Ann. 116 (1939), 1-50. MR1513216

192. N. Wiener, The ergodic theorem, Duke Math. J. 5 (1939), 1-18. MR1546100

193. T. Wolff, An improved bound for Kakeya type maximal functions, Rev. Mat. Iberoamericana 11 (1995), 651-674. MR1363209 (96m:42034)

194. T. Wolff, A Kakeya type problem for circles, Amer. J. Math. 119 (1997), 985-1026. MR1473067 (98m:42027)

195. T. Wolff, A mixed norm estimate for the X-ray transform, Rev. Mat. Iberoamericana 14 (1998), 561-601. MR1681585 (2000j:44006)

196. T. Wolff, Decay of circular means of Fourier transforms of measures, Internat. Math. Res. Notices 10 (1999), 547-567. MR1692851 (2000k:42016)

197. T. Wolff, Recent work connected with the Kakeya problem, in Prospects in Mathematics, $\mathrm{H}$. Rossi, ed., Amer. Math. Soc., Providence, R.I. (1999), 129-162. MR1660476 (2000d:42010)

198. T. Wolff, Local smoothing type estimates in $L^{p}$ for large p, Geom. Funct. Anal. 10 (2000), 1237-1288. MR1800068 (2001k:42030)

199. T. Wolff, A sharp bilinear cone restriction estimate, Ann. Math. 153 (2001), 661-698. MR1836285 (2002j:42019)

200. T. Wolff, Lectures on Harmonic Analysis, I. Łaba and C. Shubin, eds., Amer. Math. Soc., Providence, R.I. (2003). MR2003254 (2004e:42002)

201. T. Ziegler, Universal characteristic factors and Furstenberg averages, J. Amer. Math. Soc. 20 (2007), 53-97. MR2257397

Department of Mathematics, University of British Columbia, Vancouver, B.C. V6T 1Z2, CANADA

E-mail address: ilaba@math.ubc.ca 